\title{
Multiparameter evaluation of acrylamide HEMA alternative monomers in 2-
}

\section{step adhesives}

M. H. Ahmed ${ }^{1,2 *}$, K. Yoshihara ${ }^{3,4 *}$, C. Yao $^{1,5}$, Y. Okazaki ${ }^{1,6}$, K. Van Landuyt ${ }^{1}$, M. Peumans ${ }^{1}$, B. Van Meerbeek $^{1}$

${ }^{1} \mathrm{KU}$ Leuven (University of Leuven), Department of Oral Health Sciences, BIOMAT \& UZ Leuven (University Hospitals Leuven), Dentistry, Leuven, Belgium; ${ }^{2}$ Tanta University, Department of Dental Biomaterials, Tanta, Egypt; ${ }^{3}$ National Institute of Advanced Industrial Science and Technology (AIST), Health Research Institute, Kagawa, Japan; ${ }^{4}$ Okayama University, Graduate School of Medicine, Dentistry and Pharmaceutical Sciences, Department of Pathology \& Experimental Medicine, Okayama, Japan; ${ }^{5}$ The State Key Laboratory Breeding Base of Basic Science of Stomatology (Hubei-MOST) and Key Laboratory for Oral Biomedicine Ministry of Education, Wuhan, China; ${ }^{6}$ Hiroshima University, Department of Advanced Prosthodontics, Hiroshima, Japan. *Equal first-author contribution.

Corresponding author: Prof. Dr. Bart Van Meerbeek, KU Leuven, Department of Oral Health Sciences, BIOMAT, Kapucijnenvoer 7, block a - box 7001, BE-3000 Leuven, Belgium. TEL: +32-16-337587, FAX: +32-16-332752, bart.vanmeerbeek@kuleuven.be.

Short title: Acrylamide HEMA alternatives in dental adhesives.

Keywords: HEMA, Durability, Acrylamides, Linear Mixed-Effects model (LME), TEM, Bond strength

\section{Conflict of Interest:}

The authors have no commercial interests in the products hereby investigated

\section{Acknowledgment}

Kuraray Noritake is gratefully thanked for providing the composite materials employed.

\section{FUNDING}

M.H. Ahmed was appointed as PhD Research Fellow at KU Leuven - BIOMAT, based on funding provided by the Ministry of Higher Education of Egypt. This study was supported in part by the JSPS grant KAKENHI 18K17068. 


\section{Multiparameter evaluation of acrylamide HEMA alternative monomers in 2-}

\section{step adhesives}

\section{ABSTRACT}

Objectives: As frequently added to adhesives, the mono-functional monomer 2-hydroxyethyl methacrylate (HEMA) acts as co-solvent and improves surface wetting. Nevertheless, HEMA promotes watersorption and hydrolysis at adhesive interfaces, affecting bond durability to dentin. This study investigated if two acrylamide co-monomer alternatives could replace HEMA in experimental adhesive-resin formulations as part of 3/2-step universal adhesives applied, respectively, in etch-andrinse (E\&R) and self-etch (SE) bonding modes.

Methods: Upon priming dentin with the 10-MDP-based Clearfil SE Bond 2' primer ('CSE2p'; Kuraray Noritake), three experimental adhesive resins, consisting of 50wt\% Bis-GMA, 15wt\% TEGDMA, and either 35wt\% diethyl acrylamide ('DEAA'), hydroxyethyl acrylamide ('HEAA') or HEMA ('HEMA+'), were applied. The control HEMA-free adhesive resin contained 60wt\% Bis-GMA and 40wt\% TEGDMA ('HEMA-'). All adhesives were evaluated for 'immediate' and 'aged' micro-tensile bond strength ( $\mu$ TBS) to dentin upon, respectively, 1-week $(1 \mathrm{w})$ and 6 -month $(6 \mathrm{~m})$ water storage, TEM adhesive-dentin interfacial interaction, 24-hour and $6 \mathrm{~m}$ three-point bending, contact-angle wetting, viscosity and watersorption.

Results: Linear mixed-effects model statistics revealed significantly better bonding performance of the adhesives applied in E\&R than SE mode, except for DEAA_1w, with the highest $\mu$ TBSs recorded for DEAA and HEMA- applied in SE mode. In E\&R mode, aging did not significantly reduce DEAA's $\mu$ TBS. Best wetting on primed dentin was recorded for HEMA+, significantly better than DEAA, further HEAA and HEMA-, these directly related to their viscosity. HEAA absorbed significantly more water than all other adhesive-resin formulations. HEMA->DEAA>HEAA>HEMA+ was the significant order for $6 \mathrm{~m}$ bending strength.

Conclusions: The acrylamide co-monomer DEAA could replace HEMA, while HEAA not. 


\section{INTRODUCTION}

Dental adhesive technology has greatly evolved in recent years towards achieving better longevity of direct and indirect adhesive restorations with simplified application procedures. Despite being the most recent generation of dental adhesives, universal adhesives, which optionally can be applied in a self-etch (SE) or etch-and-rinse (E\&R) bonding mode, are still thought to have shortcomings with a definite need to improve their long-term bond durability [1-3]. Thus keeping conventional 3-step E\&R and (mild) 2-step SE adhesives, the latter preceded by selective enamel etching and thus also involving a 3-step application procedure, as benchmarks for reliable, predictable and durable bonding in routine clinical practice [4].

Universal adhesives provide acidic aqueous single-solution blends of hydrophilic and hydrophobic components to achieve time-stable bonding to dentin (and enamel) following both main bonding modes $[5,6]$. The mono-functional monomer 2-hydroxyethyl methacrylate (HEMA) is frequently added to many of today's commercial adhesives as an essential component. Thanks to its inherent hydrophilic nature, HEMA acts as co-solvent for other hydrophobic monomers that mix less good in water, hereby preventing phase separation between monomer ingredients and water [7]. In addition, HEMA promotes surface wetting and intra-substrate infiltration thanks to its small molecular size [8].

Nevertheless, HEMA exhibits a noticeably low degree of polymerization conversion (DC) [9]. It holds water, present as basic ingredient in dental adhesives up to $40 \%[5,10]$, inside the adhesive, by which water is more difficult to remove by gentle air-blowing upon the adhesive's application. If water remains within the adhesive, it impairs polymerization. All these factors enhance the adhesive's solubility and promote water uptake, almost immediately from the underlying dentin through osmosis and later also from the outside oral environment [11, 12]. Furthermore, HEMA has been documented to negatively inhibit Ca-nanolayering of 10-MDP at SE adhesive interfaces [13]. Impaired physicomechanical properties of the resultant polymer network at the adhesive-dentin interface promotes time-dependent hydrolytic degradation of the adhesive-dentin bond, which eventually compromises the tooth restoration's longevity [9].

Recently, acrylamide monomers have been suggested as potential candidates to replace HEMA. Their amide group $\left(\mathrm{R}_{1}-\mathrm{CO}-\mathrm{NR}_{2}-\mathrm{R}_{3}\right)$ is thought to be more stable and resistant to degradation than the ester group $\left(\mathrm{R}_{1}-\mathrm{CO}-\mathrm{O}-\mathrm{R}_{2}\right)$ of HEMA and other methacrylate monomers $[14,15]$. Despite inherently being also hydrophilic, acrylamide monomers are thought to be more hydrolytically stable than HEMA. Hydroxyethyl acrylamide (HEAA) has recently been suggested as HEMA alternative [14, 15], but solely its rheological properties were investigated, while HEAA was not yet added to an adhesive formulation. Diethyl acrylamide (DEAA) is another candidate acrylamide monomer to replace HEMA in dental adhesives. DEAA is expected to be more hydrophobic than HEAA due to the two terminal ethyl groups, but still keeps the amide group in the middle as balance. 
The objectives of this study were to investigate if HEAA and/or DEAA could successfully replace HEMA in experimental adhesive-resin formulations as part of 3/2-step universal adhesives applied, respectively, in etch-and-rinse (E\&R) and self-etch (SE) bonding modes, this following a multiparametric evaluation protocol. The null hypotheses tested were that (1) the bonding effectiveness and durability of the DEAA- and/or HEAA-containing adhesive resins, when applied to equally primed dentin, did not significantly differ from the HEMA-containing adhesive resin when employed in E\&R and SE bonding modes and upon substantial aging, (2) DEAA- and/or HEAAcontaining adhesive resins did not significantly differ from the HEMA-containing version for the different parameters of relevance evaluated, and (3) DEAA and/or HEAA can replace HEMA in $3 / 2$ step universal adhesives.

\section{MATERIALS \& METHODS}

\subsection{Experimental adhesive-resin preparation}

Four experimental adhesive-resin formulations were prepared to be applied upon priming dentin. Three adhesive resins consisted of 50wt\% Bis-GMA, 15wt\% TEGDMA, and either 35wt\% diethyl acrylamide ('DEAA'), hydroxyethyl acrylamide ('HEAA') or HEMA ('HEMA+') (Table 1). The fourth HEMA-free adhesive resin, which contained 60wt\% Bis-GMA and 40wt\% TEGDMA ('HEMA-'), served as control. The monomers BisGMA, TEGDMA and HEMA were purchased from Sigma Aldrich (St. Louis, MO, USA), while the monomers DEAA and HEAA were purchased from Tokyo Chemical Industry (Tokyo, Japan). The molecular weight and partition coefficient $(\log P)$ of all compositional monomers were determined by a free online cheminformatics database platform (Molinspiration, Molinspiration Cheminformatics, Slovensky Grob, Slovak Republic), as listed in Table 1. The molecular and 3D chemical structures of the monomers DEAA, HEAA and HEMA are shown in Fig. 1.

\subsection{Dentin-specimen preparation and adhesive application}

Thirty-two non-carious human third molars were collected (following informed consent approved by the Commission for Medical Ethics of the host institute under file number S54254) and stored in an aqueous solution of $0.5 \%$ chloramine $\mathrm{T}$ trihydrate powder at $4^{\circ} \mathrm{C}$ to prevent bacterial growth until use; all teeth were used within 3 months after extraction. The teeth were randomly divided into four groups of 8 teeth each $(n=8)$.

The occlusal third of the crowns was removed using a water-cooled slow-speed diamond saw (Isomet 1000, Buehler, Lake Bluff, IL, USA) after mounting the teeth in gypsum blocks. Next, a uniform smear layer was produced on all exposed mid-coronal dentin surfaces using a high-speed medium-grit (107 $\mu \mathrm{m})$ diamond bur (882, Komet, Lemgo, Germany) mounted in a modified Micro-Specimen Former 
(University of lowa, lowa City, IA, USA). The prepared dentin surfaces were examined for absence of enamel and/or exposure of pulp tissue using a stereomicroscope (Wild M5A, Wild Heerbrugg, Heerbrugg, Switzerland).

A split-tooth design was used with each experimental adhesive applied in E\&R mode on one tooth half and in SE mode on the other corresponding tooth half, totaling to 8 experimental groups. The flat surface was divided into two nearly equal parts by cutting a shallow $0.5-\mathrm{mm}$ deep groove using a thin 150- $\mu \mathrm{m}$ diamond blade (Isomet 1000, Buehler). Prior to 10-s phosphoric-acid etching, a single-edge carbon-steel blade (EMS, Hatfield, PA, USA) was placed in the groove between the two tooth halves in order to prevent acid leakage from the E\&R half to the SE half.

All experimental adhesives were applied and light-cured as per instructions for use of the goldstandard 2-step SE adhesive Clearfil SE Bond 2 ('C-SE2'; Kuraray Noritake, Tokyo, Japan) (Table 1). Clearfil SE Bond 2 primer ('C-SE2primer'; Kuraray Noritake) was applied strictly following its manufacturer's instructions prior to application of the experimental adhesive-resin formulations following a 2-step SE (2SE) application, and as part of a 3-step E\&R (not following manufacturer's instructions; 3E\&R), the latter upon beforehand etching with 35\% phosphoric acid (K-Etchant Syringe, Kuraray Noritake) for $10 \mathrm{~s}$, and (Table 1). Each tooth was then restored in four composite (Clearfil AP$X$, Kuraray Noritake; shade A3) increments ( $1.5 \mathrm{~mm}$ each) to achieve a 5-6 $\mathrm{mm}$ build-up height. Each increment was light-cured for $10 \mathrm{~s}$ (as per Clearfil AP-X instructions for use), with each side of the composite build-up additionally light-cured for $10 \mathrm{~s}$, so ensuring sufficient total curing time and excluding that insufficient light-curing would have affected the data. All light-curing done in this study was carried out using the LED light-curing unit Bluephase 20i (Ivoclar Vivadent, Schaan, Liechtenstein) in high mode with a power output of at least $1200 \mathrm{~mW} / \mathrm{cm}^{2}$, as confirmed by a Marc Resin Calibrator (BlueLight Analytics, Halifax, Canada).

\subsection{Micro-tensile bond-strength ( $\mu$ TBS) testing}

Following light curing, the restored teeth were stored for one week (1w) in distilled water at $37^{\circ} \mathrm{C}$. Next, the teeth were sectioned using a precision-cutting machine (Accutom 50, Struers, Ballerup, Denmark) to produce 1- $\mathrm{mm}^{2}\left( \pm 0.1 \mathrm{~mm}^{2}\right)$ sticks. All micro-specimens originating from the central tooth area (up to 24 specimens per tooth) were collected from each tooth half in order to minimize dentinal regional variability. The adhesive interfaces of all micro-specimens were examined for absence of enamel at the corners using the stereomicroscope (Wild M5A, Wild Heerbrugg). The collected microspecimens from each experimental group were then randomly distributed into two subgroups. The $\mu \mathrm{TBS}$ of half of the specimens was measured after $1 \mathrm{w}$ storage in distilled water at $37^{\circ} \mathrm{C}$, being referred to as immediate ' $1 \mathrm{w} \mu \mathrm{TBS}$ ', with the remaining half stored for 6 months $(6 \mathrm{~m})$ in distilled water at $37^{\circ} \mathrm{C}$ prior to being tested to determine the aged ' $6 \mathrm{~m} \mu \mathrm{TBS}$ '. 
The exact micro-specimen dimensions were measured using a digital caliper (DIGI-MET, Helios Preisser, Gammertingen, Germany), prior to fixing the specimens to BIOMAT jigs with cyanoacrylate glue (Model Repair II Blue, Dentsply-Sankin, Ohtawara, Japan) [16]. The non-trimmed rectangular micro-specimens were stressed in tension using a universal testing device (LRX, Lloyd, Hampshire, UK) at a crosshead speed of $1 \mathrm{~mm} / \mathrm{min}$, upon which the $\mu \mathrm{TBS}$ was calculated in MPa by dividing the force imposed at the time of fracture by the bond area $\left(\mathrm{mm}^{2}\right)$. Any pre-test failures (ptf's) during cutting, storage, aging and/or jig mounting were explicitly recorded and included by default in the statistical analysis as $0 \mathrm{MPa}$. The $\mu \mathrm{TBS}$ protocol prescribed by the Academy of Dental Materials was strictly followed [17].

Statistical analysis was carried out using linear mixed-effects statistical modelling with specific contrast ('LME'; R software) to determine statistical significance at a significance level of $\alpha$ equaling 0.05 [18]. The random factor applied in the statistical model was the individual tooth ( 32 teeth in total), with each group of sticks nested within its father tooth. Three variables were identified for the LME statistical model: 'Adhesive' with the four levels DEAA, HEAA, HEMA- and HEMA+; 'Bonding mode' with the two levels SE and E\&R; and 'Aging' with the two levels $1 \mathrm{w}$ and $6 \mathrm{~m}$. First, $2^{\text {nd }}$, and $3^{\text {rd }}$ order interactions of all variables were evaluated for statistical significance.

All fractured $\mu$ TBS micro-specimen pairs (dentin and composite side) were examined using stereomicroscopy (Wild M5A, Wild Heerbrugg) to determine the mode of failure, which was recorded either as 'mixed failure' (M), 'cohesive failure in composite' (C), 'adhesive interfacial failure' (I) or 'cohesive failure in dentin' (D). The failure mode of selected representative micro-specimen pairs (2-3 specimens/experimental group with a $\mu$ TBS close to the mean) as well as of 2-3 ptf specimens (when available) were subsequently characterized using scanning electron microscopy (SEM; JSM-6610LV, Jeol, Tokyo, Japan), upon common SEM specimen processing, including fixation, gradual dehydration, chemical drying and gold-sputter coating, this using a procedure described in detail before [19].

\subsection{Ultra-structural interfacial TEM characterization of adhesive-dentin interfaces}

TEM specimens of adhesive-dentin interface cross-sections were prepared upon application of all experimental adhesives strictly following the same methodology described above for the immediate $\mu$ TBS specimens. TEM-specimen processing was done following common procedures, including successively fixation, gradual dehydration and embedding, this according to the procedure described in detail before [20].

\subsection{Three-point bending test (flexural strength)}

Each adhesive-resin formulation was dispensed in a prefabricated Teflon mold with an inner length of $25 \mathrm{~mm}$, width of $2 \mathrm{~mm}$ and height of $2 \mathrm{~mm}$. Twenty specimens in total per experimental adhesive resin 
were fabricated. The flexural strength (FS) of half of the specimens of each group $(n=10)$ was measured after 24 -hour $(24 \mathrm{~h})$ water storage at $37^{\circ} \mathrm{C}$, while the second half was tested after $6 \mathrm{~m}$ water storage at $37^{\circ} \mathrm{C}$. FS was measured in MPa using the formula:

\section{$F S=3 F L / w h^{2}$}

with $\mathrm{F}$ being the maximum force applied, $\mathrm{L}$ the length of the supported specimen part (support span), $\mathrm{w}$ the specimen width and $\mathrm{h}$ is the specimen height of the sample. Data obtained in MPa were statistically analyzed with two-way ANOVA followed by Tukey's post-hoc test using R software [18].

\subsection{Contact-angle (CA) measurements}

Three dentin discs from three different teeth were prepared for each experimental adhesive resin (12 teeth in total; $n=3$ ), in the way as detailed before for $\mu$ TBS testing. Upon application of C-SE2 primer (Table 1), one drop of each experimental adhesive resin was deposited using a syringe with a $22 \mathrm{G}$ needle (ES004ASTF22G, Excimer.Inc, Yokohama, Japan) on the primed dentin surface. CA was measured using a CA measurement device (SImage AUTO 100, Excimer.Inc). CA data were reported in degrees and statistically analyzed with one-way ANOVA using R software [18].

\subsection{Viscosity (Vs)}

Three solutions of one-gram ethanol added to $1 \mathrm{~g}$ of each experimental adhesive resin were prepared, upon which each solution was mixed for 15 min using a stirrer. The viscosity of the obtained solutions was measured using a Tuning Fork Vibro Viscometer (SV-1A, A\&D, Tokyo, Japan) ( $n=3)$. Data obtained in $\mathrm{mPa}$.S were statistically analyzed with one-way ANOVA followed by Tukey's post-hoc test using $\mathrm{R}$ software [18].

\subsection{Watersorption (Ws)}

Samples to measure watersorption were prepared according to ISO 4049 standard. Each experimental adhesive resin was added into a Teflon mold with an inner diameter of $15 \mathrm{~mm}$ and a thickness of 1 $\mathrm{mm}$, rendering a volume $(\mathrm{V})$ of $176.6 \mathrm{~mm}^{3}$. A glass slide covered with a transparent $50-\mu \mathrm{m}$ polyester film served mold bottom. A second transparent polyester film was placed on top of each mold in tight contact with the adhesive resin. Another glass slide was placed on the film and fixed with two clamps, upon which the adhesive resin was light-cured by exposure for $40 \mathrm{~s}$ to an LED light-curing unit (G-light Prima II plus, GC, Tokyo, Japan) with an output of at least $1200 \mathrm{~mW} / \mathrm{cm}^{2}$. Three samples were prepared for each adhesive resin $(n=3)$ and weighed to reveal ' $m 1$ '. Samples were then stored in distilled water in an incubator at $37^{\circ} \mathrm{C}$ for 6 months. After 6 months, all specimens were gently rinsed with distilled water, wiped off with absorbent paper, left in the air for $15 \mathrm{~s}$ and finally weighed no later than $1 \mathrm{~min}$ 
after removal from the incubator to record their mass ' $\mathrm{m} 2$ '. The values for watersorption (Ws) were calculated for each specimen using the following formula:

$$
W s=m 2-m 1 / V
$$

Data were obtained in $\left(\mu \mathrm{g} / \mathrm{mm}^{3}\right)$ and statistically analyzed with two-way ANOVA followed by Tukey's post-hoc test using R software [18].

\section{RESULTS}

The mean $\mu$ TBS and fitted LME means are graphically represented in Figs. 2 and 3, when the experimental adhesives were applied in a 3E\&R and 2SE mode, respectively, and numerically detailed in Table 2. Statistically significant differences in $\mu$ TBS are mentioned in Figs. 2 and 3 with specific symbols and explained in the graph legend.

In the first step of the statistical analysis, a first-order interaction for each of the three study variables 'Adhesive', 'Bonding mode' and 'Aging' was individually tested. All the three study variables were found significant, indicating that each individual variable should have influenced the resulted $\mu$ TBS. Second-order interaction analysis revealed that the 'Adhesive * Bonding mode' interaction was statistically significant, indicating that the bonding mode should have affected the effect of the adhesive, or vice versa, on the resultant $\mu$ TBS. The third-order interaction, combining all three variables, was found significant and hence proposed as the final statistical model, based upon which the fitted LME means were predicted (Table 3).

Regarding the variable 'Adhesive', as compared to the HEMA+ (statistical difference indicated by ' $\mathbf{X}$ ' in Figs. 2 and 3), solely DEAA and HEMA- revealed significantly higher fitted LME means ( $\mu$ TBS) than HEMA+ when applied in SE bonding mode and tested after 1w storage. No other significant differences in immediate $\mu$ TBS with that of HEMA+ were found.

Regarding the variable 'Bonding mode', comparing the difference between E\&R and SE bonding mode (statistical difference indicated by ' $\star$ ' in Figs. 2 and 3), all the experimental adhesives revealed a significantly higher $\mu$ TBS when applied in 3E\&R than in 2SE mode, except for DEAA's immediate $\mu$ TBS.

Regarding the variable 'Aging', as compared to the $1 \mathrm{w} \mu \mathrm{TBS}$ (statistical difference indicated by '\#' in Figs. 2 and 3), a significant decrease in $\mu$ TBS after ' $6 \mathrm{~m}$ ' aging was solely recorded for HEMA- and HEMA+ when these experimental adhesives were applied in 3E\&R mode. Overall, DEAA appeared most stable, as no significant reduction in $\mu$ TBS was recorded upon aging when applied in both 3E\&R and 2SE bonding mode. Also, HEAA's $\mu$ TBS did not significantly decrease upon aging, but its $\mu$ TBSs were generally the lowest of all experimental groups, in part also related to the high number of pre-test failures (ptf's) recorded when HEAA was applied in 2SE mode. 
Fractographic analysis revealed that most specimens failed adhesively at the interface, with more or even solely 'interfacial' failures being recorded for HEAA and HEMA+, in particular when applied in SE mode (Fig. 4). DEAA on the contrary showed the most differentiated fractographic data of all experimental adhesives.

SEM of the fractured specimens disclosed the main failure modes. The examined DEAA specimens revealed relatively uniform interfacial failures, except for DEAA_1w_E\&R that presented with more mixed failures, often exhibiting fractured dentin parts that remained attached to the composite side. The other DEAA specimens showed partial or complete debonding of the adhesive layer from dentin with exposure of the hybrid layer (Figs. 5a-h). HEAA also revealed mainly interfacial and mixed failures with fractured dentin parts having remained attached in part to the composite side of the specimens (Figs. 5A-H). HEMA- and HEMA+ did not show much difference from the previous groups except for some mixed failures for HEMA+_E\&R_6m, involving partially fractured composite that remained attached to dentin (Figs. 6).

TEM interfacial characterization overall revealed tight interactions of the experimental adhesives with dentin when applied following both the 3E\&R and 2SE bonding modes (Figs. 7-10). No interface debonding was observed during specimen preparation, processing, embedding and ultra-microtomy in all experimental groups, this as an indirect indication of bond stability having been sufficient to resist the rather severe TEM-specimen preparation. As expected, the hybrid layer produced by the experimental adhesives applied in 3E\&R bonding mode is significantly thicker (3-4 $\mu \mathrm{m})$ than when produced in SE bonding mode $(<1 \mu \mathrm{m})$. E\&R hybrid layers present with clear dentin demineralization with solely few residual hydroxyapatite (HAp) remaining at the hybrid-layer bottom and a rather abrupt transition to deeper unaffected dentin; distinct resin tags were formed in the opened tubules. SE hybrid layers typically resemble the hybrid-layer ultra-morphology produced by mild SE adhesives; they disclose submicron and solely partial demineralization with abundant HAp remaining within the hybrid layer and a less abrupt transition to unaffected dentin. Intra-tubular infiltration with resin-tag formation was seldom observed in SE mode. All examined E\&R and SE TEM sections showed interfacial nano-layering near the adhesive-dentin interfaces in SE mode and at the tubule orifices when the adhesive was applied in 3E\&R mode. No major differences in interfacial ultra-morphology were detected for the different experimental adhesives when applied in the same E\&R or SE bonding mode. In general, the adhesive-layer thickness varied regionally, most likely due to local air-blowing differences, but with HEAA and HEMA+ revealing thicker adhesive-resin layers (Figs. 8 and 10: 6-8 $\mu \mathrm{m}$ ) than DEAA and HEMA-, which resulted in 3- to 4- $\mu \mathrm{m}$ thick adhesive-resin layers (Figs. 7 and 9). No voids were observed in the adhesive layer, nor at the bottom of the hybrid layer for all experimental adhesives applied in both bonding modes. 
The flexural strength (FS) of the four adhesive-resin formulations is graphically presented in Fig. 11a, with the actual mean FS (and SD) mentioned besides each column. At 24h, HEAA and HEMArevealed the significantly highest FS, HEMA+ the significantly lowest FS with an intermediate FS recorded for DEAA. After 6-month aging, the most stable FS was measured for DEAA and HEMA-, with the latter FS remaining significantly higher. HEAA's FS significantly dropped upon aging, while HEMA+'s FS remained significantly the lowest.

Applying the experimental adhesive resins on primed dentin disks revealed the significantly highest contact angles (CAs) for HEAA and HEMA-, basically revealing the lowest wetting ability (Fig. 11b). Both mean CAs were significantly higher than that of DEAA, which on its turn was significantly higher than HEMA+'s CA; HEMA+ wetted primed dentin best (Fig. 11b).

The viscosity (Vs) data closely resembled the CA-data order (Fig. 11c). HEAA and HEMA- revealed a significantly higher viscosity than DEAA and HEMA+, without significant difference between the two adhesives within each adhesive-resin pair. This difference in viscosity between the two respective adhesive-resin pairs was reflected in the difference in thickness of the adhesive-resin layers observed by TEM.

The significantly highest watersorption (Ws) was measured for HEAA, the significantly lowest for HEMA- with an intermediate but still a relatively low Ws recorded for DEAA and HEMA+ (Fig. 11d).

\section{DISCUSSION}

Most of today's commercially available dental adhesives are able to effectively bond to 'model' dentin at short term, as evidenced by a favorable 'immediate' bonding effectiveness recorded in laboratory research [21, 22]. While bond strength upon long-term aging is more frequently measured, both shortand long-term clinical effectiveness data of dental adhesives remain scarce. Especially regarding the development of adhesives with simplified application protocols, profound bond-strength studies remain therefore highly needed, as such adhesives often trade off bond durability against ease of use.

Most resin-based dental materials contain Bis-GMA as base monomer. Bis-GMA is well-known for its high hydrophobicity, adequate cure (DC) and for providing mechanical strength to the resultant polymer network [23]. Despite being a major constituent, its high viscosity necessitates the addition of diluent monomers to achieve adequate fluidity dental adhesives require [23]. One of the main functions of HEMA is to act as diluent for Bis-GMA and to improve the adhesive's wetting properties, hereby also facilitating infiltration of the resultant primer/adhesive blend into the completely (E\&R) or partially (SE) demineralized dentin surface. It is a long-known fact that HEMA improves the (immediate) bond strength of dental adhesives to dentin [9]. Nevertheless, as mentioned above, HEMA has besides pros also significant cons, among which enhanced watersorption in light of the most 
important hydrolytic bond-degradation process should be regarded as a non-neglectable drawback. Universal adhesives contain water in need for the SE bonding mode, by which HEMA is still often added as co-solvent to UAs $[1,10,24]$. Searching for adequate HEMA-replacement monomers has already appeared challenging, being also the aim of this explorative research. In today's adhesives, manufacturers attempt to substantially reduce the HEMA content [3] or even to replace HEMA by alternative monomers like methacrylamide monomer variants [25].

Indeed, the high hydrophilicity of HEMA due to its hydroxyl group at the end of the short monomer leads to increased water uptake and high solubility after polymerization $[26,27]$, thus weakening the mechanical properties of the adhesive's polymer network and jeopardizing the long-term stability of the formed bond to dentin due to hydrolytic degradation [28, 29]. HEMA as a methacrylic acid (MAA)based monomer is an ester, which makes it vulnerable to degradation by esterases [30, 31]. It then degrades into ethylene glycol and methacrylic acid, which in addition to dramatically affecting the integrity of the bonded interface, participates greatly in the genotoxicity effect of HEMA [32].

Acrylamide monomers were suggested as potential HEMA alternatives for resin-based dental adhesives and restorative materials $[14,15,33,34]$. The amide group is generally more resistant to hydrolytic degradation, as compared to the ester group in MAA-based monomers, due to the lower reactivity of the carbonyl group, this except for extreme conditions, such as in case of a highly acidic $(\mathrm{pH}<0.94)$ environment $[24,35]$. Furthermore, the nitrogen atom in amides, as compared to the oxygen atom in MAA-based monomers, tends to reduce the monomer's susceptibility to hydrolysis. This should be attributed to the lower electronegativity of nitrogen compared to oxygen, which makes it more likely to donate non-bonded electrons to the carbonyl carbon, hereby shortening and strengthening the chemical binding, and ultimately decreasing the amide's susceptibility to hydrolysis [36]. Interestingly, acrylamides were also suggested to exhibit hydrogen binding with collagen fibrils as an additional advantage for biomechanical compatibility over methacrylates [37, 38]. On top of that, the degree of conversion of acrylamide monomers was found to be higher than that of MAA-based monomers when tested as homo-monomers [14]. In line with the abovementioned reasoning, several studies have tested the potential applicability of acrylamide co-monomers as HEMA alternatives [14, 15, 33]. Although they evaluated physico-mechanical, rheological and bonding properties, these adhesives were not applied as UAs in the two main bonding modes, nor subjected to substantial aging.

In this study, we formulated three experimental adhesive resins that differed solely for the monofunctional monomer, being HEMA or one of the two experimental acrylamide HEMA alternatives DEAA and HEAA. These mono-functional monomers were added in a concentration of $35 \mathrm{wt} \%$ to a conventional 50/15wt\% Bis-GMA/TEGDMA composition. A HEMA-free adhesive resin (HEMA-) solely contained 60wt\% Bis-GMA and 40wt\% TEGDMA. All other non-monomer ingredients were equal for all four experimental adhesive resins investigated, thus enabling a direct mutual comparative analysis 
of adhesive performance by the acrylamide DEAA and HEAA formulations versus the positive control HEMA+, and all three experimental adhesive-resin formulations versus the negative control HEMA-. No solvent was added to the experimental adhesive-resin formulations in order to optimize polymerization, this in part also based on previous research that demonstrated that the stability of adhesive-dentin interfaces benefited from an extra hydrophobic bonding layer applied on top of universal or one-step (SE) adhesives with simplified application protocols $[1,2,39]$. Extensive explorative pilot testing preceded the actual experiment conducted in this study, during which the composition of the experimental adhesive-resin formulations was optimized. We opted for a universal bonding approach with the four experimental adhesives bonded to dentin in both E\&R and SE bonding modes. All adhesive-resin formulations were applied following application of the 10-MDP-based primer of the considered gold-standard adhesive Clearfil SE Bond 2 (Kuraray Noritake). Besides primary bonding performance, the experimental adhesives were tested for different parameters thought of being important for bond durability.

The $\mu$ TBS study design, involving the three variables 'Adhesive' (with the four levels DEAA, HEAA, HEMA- and HEMA+), 'Bonding mode' (with the two levels E\&R and SE), and 'Aging' (with the two levels $1 \mathrm{w}$ and $6 \mathrm{~m}$ water storage) required an LME statistical model, taking into account the split-tooth design (E\&R versus $\mathrm{SE}$ ) and enabling comparison of inter-tooth and intra-teeth differences, thus to predict proper fitted LME means [40]. Other statistical analytic methods (e.g. ANOVA, general linear model) would have decreased the power of the statistical analysis.

Overall, considering the E\&R and SE $\mu$ TBS measured upon $1 \mathrm{w}$ and $6 \mathrm{~m}$ water storage altogether, the acrylamide monomer DEAA outperformed HEAA and HEMA+, but not HEMA-. As $\mu$ TBS should be regarded as testing the strength of the total adhesive assembly, the better adhesive performance of DEAA and HEMA- is also reflected in their flexural strengths (FSs), being significantly better and more stable upon aging than those of HEAA and HEMA+. In this respect, the significantly higher FS of HEMAthan that of DEAA should be ascribed in the first place to the higher Bis-GMA concentration (+10wt\%).

The dimethacrylate monomer Bis-GMA is well known to render resin-based dental materials higher strength, less polymerization shrinkage (compared to lower MW monomers) and higher stiffness [41, 42]. However, the inherent higher viscosity of BisGMA impairs its diffusion properties and thus necessitates the use of diluent dimethacrylate monomer (f.i. TEGDMA) in order to deal with the high viscosity [41]. The low-viscosity hydrophilic HEMA co-monomer is commonly added to adhesives to additionally decrease viscosity, but mainly to promote diffusion into the dentin substrate $[43,44]$. Despite this bond-promoting effect of HEMA, there are sufficient shortcomings arising from employing HEMA in dental adhesives to justify searching for suitable HEMA alternatives. In addition, BisGMA served as base monomer in the experimental adhesive-resin formulations that were investigated in the current research, this in order to maintain BisGMA's benefits but also the study's focus on 
evaluating the acrylamide HEMA alternatives. Fugolin et al. showed in 2019 that monomer mixtures with higher Bis-GMA content could result in better mechanical properties than pure acrylamide mixtures in wet conditions [36]. Otherwise, considering the reduction percentage in FS for the four experimental formulations, it ranged from $-1.7 \%$ for DEAA, $-13.9 \%$ for HEMA-, $-20.5 \%$ for HEMA+ with the highest reduction of $-44.49 \%$ having been recorded for HEAA. If the $6 \mathrm{~m}$-aged FS data would be extrapolated to $1 y$-aged FS data (assuming the kinetics remain constant), HEMA-'s FS could have dropped below the extrapolated $1 y$-aged FS of DEAA. DEAA revealed intermediary wetting ability on primed dentin, not as good as HEMA+ but significantly better than HEAA and HEMA-. This is, in part, also related to DEAA's relatively low viscosity (Vs), as low as that of HEMA+ but significantly less viscous than that of HEAA and HEMA-. Besides promoting wetting, DEAA's lower viscosity must have helped this acrylamide monomer to more effectively hybridize dentin. DEAA also revealed low watersorption (Ws), significantly lower than that of HEAA and only slightly more than that of the most hydrophobic adhesive resin HEMA-.

Significantly worse bonding performance was recorded for the second acrylamide monomer HEAA, when the respective experimental adhesive was applied both in 3E\&R and 2SE bonding mode. Noticeable is the relatively high ptf number as another indication of inferior bonding effectiveness. In particular, the high WS of the HEAA-based adhesive resin is detrimental to bond stability, while the relatively low wetting ability (high CA) and relatively high Vs may also have hindered hybridization [45, 46]. Comparing both acrylamide monomers, the hydroxyl group makes HEAA much more hydrophilic than DEAA (possessing two ethyl groups), explaining HEAA's higher Ws and resultant aging-sensitive FS and inferior $\mu$ TBS. The higher hydrophilic nature of HEAA might have prevented complete water removal by gentle air-blowing upon adhesive-resin application, which could in turn have impaired polymerization and the developed mechanical properties, a scenario similar to that documented for HEMA $[5,10]$. Less expected was the adhesive performance of HEMA-, which without containing a monofunctional monomer performed as good as DEAA for $\mu$ TBS and better than DEAA for FS. HEMA's wetting ability on primed dentin was the lowest of all adhesive-resin formulations investigated, but this adhesive resin absorbed the significantly least water. Except for the significantly lowest FS, HEMA+ performed overall quite well for the different parameters evaluated.

Overall, all three null hypotheses tested in this study were accepted for the alternative co-monomer HEAA but failed to be accepted for DEAA. In this respect, the monomer partition coefficient (logP) is a good indicator of monomer hydrophilicity (Table 1). LogP is determined by measuring the concentration ratio of a compound in a mixture of two immiscible phases/solvents (most commonly water and octan-1-ol) at equilibrium [47]. Various experimental methods are valid to measure LogP, including chemical software as was employed in the current study. It was not possible to measure the overall $\log \mathrm{P}$ of the adhesive-resin formulations as a blend. However, since all the formulated adhesive 
resins were identical in composition except for HEMA and the acrylamide alternatives, the logP value determined for the individual monomers could be a good indicator of that of the respective adhesiveresin formulation. HEAA presented with the lowest $\log \mathrm{P}(-0.57)$ and the lowest molecular weight (115.13), indicating its relatively high and more-than-needed hydrophilicity, which must be the main reason for the high Ws recorded and for a great part also be responsible for HEAA's overall recorded inferior performance. On the other hand, although DEAA presented with a similarly low molecular weight (127.19), its higher $\log P(1.06)$ indicated that DEAA's nature was more hydrophobic. The difference in molecular weight between the acrylamide monomers investigated and HEMA (130.1) was relatively small. However, the three monomers presented with a substantial difference in logP, with the highest logP recorded for DEAA, indicating to be most hydrophobic. This reasoning supports the lower Ws recorded for DEAA and its overall recorded better performance than HEAA and HEMA. Indeed, Dickens et al. [48] concluded that logP is a good predictor of watersorption; it highlights the complexity of relationships between structure and properties of polymer networks $[36,49]$.

\section{CONCLUSION}

This study investigated the potential applicability of two acrylamide monomers, namely DEAA (diethyl acrylamide) and HEAA (hydroxyethyl acrylamide), to replace HEMA (hydroxyethyl methacrylate) in adhesive-resin formulations. Considering all parameters evaluated, DEAA outperformed HEAA, by which it is concluded that DEAA could potentially replace HEMA, but HEAA not. 


\section{REFERENCES}

[1] Ahmed MH, De Munck J, Van Landuyt K, Peumans M, Yoshihara K, Van Meerbeek B. Do universal adhesives benefit from an extra bonding layer? J Adhes Dent. 2019;21:117-32. https://doi.org/10.3290/j.jad.a42304.

[2] Ermis RB, Ugurlu M, Ahmed MH, Van Meerbeek B. Universal adhesives benefit from an extra hydrophobic adhesive layer When light cured beforehand. J Adhes Dent. 2019;21:179-88. https://doi.org/10.3290/j.jad.a42344.

[3] Ahmed MH, Yoshihara K, Mercelis B, Van Landuyt K, Peumans M, Van Meerbeek B. Quick bonding using a universal adhesive. Clin Oral Investiig. 2019:1-15. https://doi.org/10.1007/s00784-019-03149$\underline{8}$.

[4] Van Meerbeek B, Peumans M, Poitevin A, Mine A, Van Ende A, Neves A, et al. Relationship between bond-strength tests and clinical outcomes. Dent Mater. 2010;26:100-21. https://doi.org/10.1016/j.dental.2009.11.148.

[5] Moszner N, Salz U, Zimmermann J. Chemical aspects of self-etching enamel-dentin adhesives: a systematic review. Dent Mater. 2005;21:895-910. https://doi.org/10.1016/i.dental.2005.05.001.

[6] Van Landuyt KL, Snauwaert J, De Munck J, Peurnans M, Yoshida Y, Poitevin A, et al. Systematic review of the chemical composition of contemporary dental adhesives. Biomaterials. 2007;28:375785. https://doi.org/10.1016/j.biomaterials.2007.04.044.

[7] Spencer P, Wang Y. Adhesive phase separation at the dentin interface under wet bonding conditions. J Biomed Mater Res. 2002;62:447-56. https://doi.org/10.1002/jbm.10364.

[8] Nakabayashi N, Takarada K. Effect of HEMA on bonding to dentin. Dent Mater. 1992;8:125-30. https://doi.org/10.1016/0109-5641(92)90067-M.

[9] Van Landuyt KL, Snauwaert J, Peumans M, De Munck J, Lambrechts P, Van Meerbeek B. The role of HEMA in one-step self-etch adhesives. Dent Mater. 2008;24:1412-9. https://doi.org/10.1016/j.dental.2008.02.018.

[10] Salz U, Zimmermann J, Zeuner F, Moszner N. Hydrolytic stability of self-etching adhesive systems. J Adhes Dent. 2005;7:107-16. https://doi.org/10.3290/j.jad.a10282.

[11] Van Landuyt KL, Snauwaert J, De Munck J, Coutinho E, Poitevin A, Yoshida Y, et al. Origin of interfacial droplets with one-step adhesives. J Dent Res. 2007;86:739-44. https://doi.org/10.1177/154405910708600810.

[12] Tay FR, Pashley DH, Suh BI, Carvalho RM, Itthagarun A. Single-step adhesives are permeable membranes. J Dent. 2002;30:371-82. https://doi.org/10.1016/S0300-5712(02)00064-7.

[13] Yoshida Y, Yoshihara K, Hayakawa S, Nagaoka N, Okihara T, Matsumoto T, et al. HEMA inhibits interfacial Nano-layering of the functional monomer MDP. J Dent Res. 2012;91:1060-5. https://doi.org/10.1177/0022034512460396. 
[14] Rodrigues SB, Collares FM, Leitune VCB, Schneider LFJ, Ogliari FA, Petzhold CL, et al. Influence of hydroxyethyl acrylamide addition to dental adhesive resin. Dent Mater. 2015;31:1579-86. https://doi.org/10.1016/i.dental.2015.10.005.

[15] Rodrigues SB, Petzhold CL, Gamba D, Leitune VCB, Collares FM. Acrylamides and methacrylamides as alternative monomers for dental adhesives. Dent Mater. 2018;34:1634-44. https://doi.org/10.1016/i.dental.2018.08.296.

[16] Poitevin A, De Munck J, Van Landuyt K, Coutinho E, Peumans M, Lambrechts P, et al. Influence of three specimen fixation modes on the micro-tensile bond strength of adhesives to dentin. Dent Mater J. 2007;26:694-9. https://doi.org/10.4012/dmj.26.694.

[17] Armstrong S, Breschi L, Özcan M, Pfefferkorn F, Ferrari M, Van Meerbeek B. Academy of Dental Materials guidance on in vitro testing of dental composite bonding effectiveness to dentin/enamel using micro-tensile bond strength ( $\mu$ TBS) approach. Dent Mater. 2017;33:133-43. https://doi.org/10.1016/i.dental.2016.11.015.

[18] R Core Team. R: A language and environment for statistical computing. Vienna, Austria: R Foundation for Statistical Computing; 2019.

[19] Perdigao J, Lambrechts P, Van Meerbeek B, Vanherle G, Lopes AL. Field emission SEM comparison of four postfixation drying techniques for human dentin. J Biomed Mater Res. 1995;29:1111-20. https://doi.org/10.1002/jbm.820290911.

[20] Van Meerbeek B, Yoshida Y, Lambrechts P, Vanherle G, Duke ES, Eick JD, et al. A TEM study of two water-based adhesive systems bonded to dry and wet dentin. J Dent Res. 1998;77:50-9. https://doi.org/10.1177/00220345980770010501.

[21] Chen C, Niu LN, Xie H, Zhang ZY, Zhou LQ, Jiao K, et al. Bonding of universal adhesives to dentineold wine in new bottles? J Dent. 2015;43:525-36. https://doi.org/10.1016/j.jdent.2015.03.004.

[22] De Munck J, Mine A, Poitevin A, Van Ende A, Cardoso MV, Van Landuyt KL, et al. Meta-analytical review of parameters involved in dentin bonding. J Dent Res. 2012;91:351-7. https://doi.org/10.1177/0022034511431251.

[23] MacAulay M, Tam L, Santerre J, Finer Y. In vivo biodegradation of bisGMA and urethane-modified bisGMA-based resin composite materials. JDR Clinical \& Translational Research. 2017;2:397-405. https://doi.org/10.1177/2380084417722117.

[24] Moszner N, Salz U. Recent developments of new components for dental adhesives and composites. Macromol Mater Eng. 2007;292:245-71. https://doi.org/10.1002/mame.200600414.

[25] Van Meerbeek B, Yoshihara K, Van Landuyt K, Yoshida Y, Peumans M. From Buonocore's pioneering acid-etch technique to self-adhering restoratives. A status perspective of rapidly advancing dental adhesive technology. J Adhes Dent. 2020;22:7-34. https://doi.org/10.3290/i.jad.a43994. 
[26] Malacarne J, Carvalho RM, de Goes MF, Svizero N, Pashley DH, Tay FR, et al. Water sorption/solubility of dental adhesive resins. Dent Mater. 2006;22:973-80. https://doi.org/10.1016/j.dental.2005.11.020.

[27] Ito S, Hashimoto M, Wadgaonkar B, Svizero N, Carvalho RM, Yiu C, et al. Effects of resin hydrophilicity on water sorption and changes in modulus of elasticity. Biomaterials. 2005;26:6449-59. https://doi.org/10.1016/i.biomaterials.2005.04.052.

[28] Hashimoto M, Fujita S, Kaga M, Yawaka Y. In vitro durability of one-bottle resin adhesives bonded to dentin. Dent Mater J. 2007;26:677-86. https://doi.org/10.4012/dmj.26.677.

[29] Torkabadi S, Nakajima M, Ikeda M, Foxton RM, Tagami J. Bonding durability of HEMA-free and HEMA-containing one-step adhesives to dentine surrounded by bonded enamel. J Dent. 2008;36:806. https://doi.org/10.1016/i.jdent.2007.11.001.

[30] Jung YJ, Hyun HK, Kim YJ, Jang KT. Effect of collagenase and esterase on resin-dentin interface: a comparative study between a total-etch adhesive and a self-etch adhesive. Am J Dent. 2009;22:295-8. [31] Pashley DH, Tay FR, Yiu C, Hashimoto M, Breschi L, Carvalho RM, et al. Collagen degradation by host-derived enzymes during aging. J Dent Res. 2004;83:216-21. https://doi.org/10.1177/154405910408300306.

[32] Szczepanska J, Poplawski T, Synowiec E, Pawlowska E, Chojnacki CJ, Chojnacki J, et al. 2Hydroxylethyl methacrylate (HEMA), a tooth restoration component, exerts its genotoxic effects in human gingival fibroblasts trough methacrylic acid, an immediate product of its degradation. Mol Biol Rep. 2012;39:1561-74. https://doi.org/10.1007/s11033-011-0895-y.

[33] Moszner N, Fischer UK, Angermann J, Rheinberger V. Bis-(acrylamide)s as new cross-linkers for resin-based composite restoratives. Dent Mater. 2006;22:1157-62. https://doi.org/10.1016/j.dental.2005.11.032.

[34] Xu XM, Wang YP, Liao SM, Wen ZZT, Fan YW. Synthesis and characterization of antibacterial dental monomers and composites. J Biomed Mater Res B. 2012;100b:1151-62. https://doi.org/10.1002/jbm.b.32683.

[35] Pei Y, Zhao L, Du G, Li N, Xu K, Yang H. Investigation of the degradation and stability of acrylamidebased polymers in acid solution: Functional monomer modified polyacrylamide. Petroleum. 2016;2:399-407. https://doi.org/10.1016/i.pet/m.2016.08.006.

[36] Fugolin AP, Dobson A, Mbiya W, Navarro O, Ferracane JL, Pfeifer CS. Use of (meth)acrylamides as alternative monomers in dental adhesive systems. Dent Mater. 2019;35:686-96. https://doi.org/10.1016/j.dental.2019.02.012.

[37] Torii Y, Itou K, Nishitani Y, Yoshiyama M, Ishikawa K, Suzuki K. Effect of self-etching primer containing $\mathrm{N}$-acryloyl aspartic acid on enamel adhesion. Dent Mater. 2003;19:253-8. https://doi.org/10.1016/S0109-5641(02)00028-3. 
[38] Nishiyama N, Suzuki K, Asakura T, Komatsu K, Nemoto K. Adhesion of N-methacryloyl-omegaamino acid primers to collagen analyzed by 13C NMR. J Dent Res. 2001;80:855-9. https://doi.org/10.1177/00220345010800030201.

[39] Van Landuyt KL, Mine A, De Munck J, Jaecques S, Peumans M, Lambrechts P, et al. Are one-step adhesives easier to use and better performing? Multifactorial assessment of contemporary one-step self-etching adhesives. J Adhes Dent. 2009;11:175-90. https://doi.org/10.4012/dmj.24.1.

[40] Minamino T, Mine A, Shintani A, Higashi M, Kawaguchi-Uemura A, Kabetani T, et al. Advanced statistical analyses to reduce inconsistency of bond strength data. J Dent Res. 2017;96:1400-5. https://doi.org/10.1177/0022034517725691.

[41] Kumar SR, Patnaik A, Bhat I. Physical and thermo-mechanical characterizations of resin-based dental composite reinforced with silane-modified nanoalumina filler particle. P I Mech Eng I-J Mat. 2016;230:504-14. https://doi.org/10.1177/1464420715581004.

[42] Sideridou I, Tserki V, Papanastasiou G. Effect of chemical structure on degree of conversion in light-cured dimethacrylate-based dental resins. Biomaterials. 2002;23:1819-29. https://doi.org/10.1016/s0142-9612(01)00308-8.

[43] Moszner N, Zeuner F, Fischer UK, Rheinberger V. Monomers for adhesive polymers, 2. Synthesis and radical polymerisation of hydrolytically stable acrylic phosphonic acids. Macromol Chem Phys. 1999;200:1062-7. $\quad$ https://doi.org/10.1002/(SICI)1521-3935(19990501)200:5<1062::AIDMACP1062>3.0.CO;2-\%23.

[44] Moszner N, Zeuner F, Angermann J, Fischer UK, Rheinberger V. Monomers for adhesive polymers, 4. Macromol Mater Eng. 2003;288:621-8. https://doi.org/10.1002/mame.200350003.

[45] Pazinatto FB, Marquezini L, Jr., Atta MT. Influence of temperature on the spreading velocity of simplified-step adhesive systems. J Esthet Restor Dent. 2006;18:38-45; discussion 6. https://doi.org/10.2310/6130.2006.00009.

[46] Sundfeld RH, da Silva AM, Croll TP, de Oliveira CH, Briso AL, de Alexandre RS, et al. The effect of temperature on self-etching adhesive penetration. Compend Contin Educ Dent. 2006;27:552-6; quiz 7, 81.

[47] Kwon Y. Handbook of essential pharmacokinetics, pharmacodynamics and drug metabolism for industrial scientists: Springer Science \& Business Media; 2002.

[48] Dickens SH, Flaim GM, Floyd CJE. Effects of adhesive, base and diluent monomers on water sorption and conversion of experimental resins. Dent Mater. 2010;26:675-81. https://doi.org/10.1016/i.dental.2010.03.011.

[49] Soman S, Chacko AS, Prasad VS. Semi-interpenetrating network composites of poly (lactic acid) with cis-9-octadecenylamine modified cellulose-nanofibers from Areca catechu husk. Compos Sci Technol. 2017;141:65-73. https://doi.org/10.1016/j.compscitech.2017.01.007. 
TABLES:

Table 1. Materials used in this study.

\begin{tabular}{|c|c|c|c|c|}
\hline Materials & Composition $^{1}$ & Mw & $\log P$ & Application procedure \\
\hline DEAA & $\begin{array}{l}\text { 35wt\% DEAA, 50wt\% Bis-GMA, 15wt\% TEGDMA } \\
\text { PLUS 1wt\% CQ (photo-initiator), 1wt\% amine } \\
\text { (co-initiator), 0.6wt\% BHT (inhibitor), 0.6wt\% 4- } \\
\text { methoxyphenol (inhibitor) }\end{array}$ & $\begin{array}{l}\text { DEAA: } \\
127.19\end{array}$ & $\begin{array}{l}\text { DEAA: } \\
1.06\end{array}$ & \multirow{4}{*}{$\begin{array}{l}\text { 3E\&R: Dentin was first etched using K-Etchant Syringe (Kuraray } \\
\text { Noritake, Tokyo, Japan) for } 10 \mathrm{~s} \text {, thoroughly water-rinsed (>10 s) } \\
\text { and gently air-dried; Clearfil SE Bond } 2 \text { primer (Kuraray Noritake) } \\
\text { was next applied as per manufacturer's instructions (see below); } \\
\text { finally, the experimental adhesive resin was applied to the entire } \\
\text { dentin surface, followed by gentle air-drying to produce a uniform } \\
\text { layer and light-curing for } 10 \mathrm{~s} \text {. } \\
\text { 2SE: Clearfil SE Bond } 2 \text { primer (Kuraray Noritake) was applied as per } \\
\text { manufacturer's instructions (see below), upon which the } \\
\text { experimental adhesive resin was applied to the entire dentin } \\
\text { surface, followed by gentle air-drying to produce a uniform layer } \\
\text { and light-curing for } 10 \mathrm{~s} \text {. }\end{array}$} \\
\hline HEAA & $\begin{array}{l}\text { 35wt\% HEAA, 50wt\% Bis-GMA, 15wt\% TEGDMA } \\
\text { PLUS 1wt\% CQ, 1wt\% amine, 0.6wt\% BHT, } \\
\text { 0.6wt\% 4-methoxyphenol }\end{array}$ & $\begin{array}{l}\text { HEAA: } \\
\quad 115.13\end{array}$ & $\begin{array}{l}\text { HEAA: } \\
\quad-0.57\end{array}$ & \\
\hline HEMA+ & $\begin{array}{l}\text { 35wt\% HEMA, 50wt\% Bis-GMA, 15wt\% TEGDMA } \\
\text { PLUS 1wt\% CQ, 1wt\% amine, 0.6wt\% BHT, } \\
\text { 0.6wt\% 4-methoxyphenol }\end{array}$ & $\begin{array}{l}\text { HEMA: } \\
\quad 130.14\end{array}$ & $\begin{array}{l}\text { HEMA: } \\
0.64\end{array}$ & \\
\hline HEMA- & $\begin{array}{l}\text { 60wt\% Bis-GMA, 40wt\% TEGDMA } \\
\text { PLUS 1wt\% CQ, 1wt\% amine, 0.6wt\% BHT, } \\
\text { 0.6wt\% 4-methoxyphenol }\end{array}$ & $\begin{array}{l}\text { Bis-GMA: } \\
\text { 512.6 } \\
\text { TEGDMA: } \\
286.32\end{array}$ & $\begin{array}{l}\text { Bis-GMA: } \\
5.26 \\
\text { TEGDMA: } \\
1.82\end{array}$ & \\
\hline $\begin{array}{l}\text { C-SE2 primer } \\
\text { (Kuraray Noritake; } \\
\text { lot 1D0057) }\end{array}$ & $\begin{array}{l}\text { 10-MDP, HEMA, hydrophilic dimethacrylate, } \\
\text { photo-initiator, water }\end{array}$ & & & $\begin{array}{l}\text { Applied as per manufacturer's instructions for use by applying the } \\
\text { primer to the entire dentin surface, then leaving undisturbed for } \\
20 \mathrm{~s} \text {, followed by gentle air-drying to help evaporating the primer } \\
\text { solvent, hereby achieving a glossy surface with the resin film not } \\
\text { visually moving anymore. }\end{array}$ \\
\hline $\begin{array}{l}\text { Clearfil AP-X } \\
\text { (Kuraray Noritake; } \\
\text { lot CL0703) }\end{array}$ & $\begin{array}{l}\text { Bis-GMA }(<12 \%) \text { TEGDMA }(<5 \%) \text {, silanated } \\
\text { barium glass filler, silanated silica filler, silanated } \\
\text { colloidal silica, dl-camphorquinone, catalysts, } \\
\text { accelerators, pigments }\end{array}$ & & & $\begin{array}{l}\text { One full compule was applied per increment to reach a } 1.5-\mathrm{mm} \text { thick } \\
\text { increment, which was immediately light-cured for } 10 \mathrm{~s} \text {; in total, } 4 \\
\text { increments were applied to reach a } 6-\mathrm{mm} \text { height; the composite } \\
\text { build-up was additionally light-cured for } 10 \mathrm{~s} \text { from each side. }\end{array}$ \\
\hline
\end{tabular}

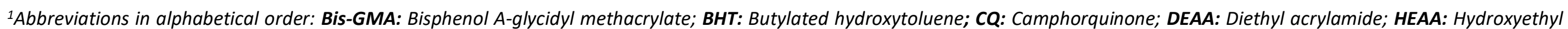
acrylamide; HEMA: 2-Hydroxyethyl methacrylate; LogP: Partition Coefficient; Mw: Molecular weight; 10-MDP: 10-Methacryloyloxydecyl dihydrogenphosphate. 
Table 2. Mean micro-tensile bond strength ( $\mu$ TBS) and fitted LME means in MPa for the different 3E\&R and 2SE experimental groups.

\begin{tabular}{rcccc}
\hline & \multicolumn{2}{c}{ IMMEDIATE (1-week) $\boldsymbol{\mu T B S}_{\text {Experimental group }}$} & \multicolumn{2}{c}{ AGED (6-month) $\boldsymbol{\mu T B S}$} \\
& Mean $\pm \mathbf{S D}^{1}\left(\mathbf{p t f ^ { 2 }} \mathbf{n}^{\mathbf{3}}\right)$ & Fitted LME mean & Mean \pm SD (ptf/n) & Fitted LME mean \\
\hline DEAA_E\&R & $24.5 \pm 15.6(6 / 42)$ & 24.8 & $26.9 \pm 15.4(6 / 42)$ & 27.3 \\
HEAA_E\&R & $21.0 \pm 14.5(1 / 29)$ & 21.0 & $18.3 \pm 11.8(5 / 33)$ & 19.0 \\
HEMA+_E\&R & $29.8 \pm 11.8(1 / 39)$ & 29.4 & $22.0 \pm 11.8(2 / 35)$ & 21.8 \\
HEMA-_E\&R & $33.2 \pm 15.6(0 / 38)$ & 33.0 & $25.3 \pm 15.2(1 / 40)$ & 34.3 \\
DEAA_SE & $19.5 \pm 13.0(6 / 41)$ & 19.4 & $16.1 \pm 8.7(4 / 41)$ & 15.5 \\
HEAA_SE & $06.2 \pm 9.3(18 / 28)$ & 06.3 & $9.5 \pm 8.7(11 / 28)$ & 09.1 \\
HEMA+_SE & $09.4 \pm 08.5(9 / 40)$ & 64.1 & $9.9 \pm 5.5(3 / 39)$ & 10.1 \\
HEMA-_SE & $19.6 \pm 10.2(3 / 34)$ & 19.7 & $17.2 \pm 10.4(2 / 39)$ & 17.0 \\
\hline
\end{tabular}

${ }^{1} \mathrm{SD}=$ Standard deviation; ${ }^{2} \mathrm{ptf}=$ Pre-test failure considered as $0 \mathrm{MPa} ;{ }^{3} n=$ Number of micro-specimens tested . 
Table 3. Linear mixed-effects (LME) statistical analysis for the first, second and third order interactions.

\begin{tabular}{lcccc}
\hline Variables & numDF $^{1}$ & denDF $^{2}$ & $\mathrm{~F}^{3}$-value & p-value \\
\hline Intercept & 1 & 544 & 354.0370 & $<.0001^{*}$ \\
Adhesive & 3 & 28 & 4.3032 & $0.0129^{*}$ \\
Bonding mode & 1 & 544 & 165.5836 & $<.0001^{*}$ \\
Aging & 1 & 544 & 6.6245 & $0.0103^{*}$ \\
Adhesive * Bonding mode & 3 & 544 & 3.0232 & $0.0293^{*}$ \\
Adhesive * Aging & 3 & 544 & 1.8537 & 0.1364 \\
Bonding mode * Aging & 1 & 544 & 2.0249 & 0.1553 \\
Adhesive * Bonding mode * Aging & 3 & 544 & 3.3925 & $0.0178^{*}$ \\
\hline
\end{tabular}

${ }^{1}$ Number of degrees of freedom; ${ }^{2}$ Denominator degrees of freedom; ${ }^{3}$ Fisher; ${ }^{*}$ Statistically significant $(p<0.05)$. 


\section{Figure captions}

Fig. 1 Schematic explaining the molecular and 3D chemical structures of the monomers investigated.

Fig. 2 Box-whisker plots (min-[lower quartile-median-upper quartile]-max) of $\mu$ TBS (in MPa) of the experimental adhesives, which contain diethyl acrylamide ('DEAA'), hydroxyethyl acrylamide ('HEAA') or 2-hydroxyethylmethacrylate ('HEMA+'), or are HEMA-free ('HEMA-'), to dentin, either measured at 1 week $(1 \mathrm{w})$ or upon 6 -month $(6 \mathrm{~m})$ water storage, this when the adhesives were applied in 3E\&R mode. The black closed dots and the red open squares represent the mean $\mu$ TBS and the fitted linear mixedeffects (LME) means, respectively. The blue thick and orange horizontal lines within each box represents the $1 \mathrm{w}$ and $6 \mathrm{~m} \mu \mathrm{TBS}$ median, respectively. Statistically significant differences between experimental groups according to the LME model are indicated by specific symbols and explained in the graph legend at the top-right side.

Fig. 3 Box-whisker plots (min-[lower quartile-median-upper quartile]-max) of $\mu$ TBS (in MPa) of the experimental adhesives, which contain diethyl acrylamide ('DEAA'), hydroxyethyl acrylamide ('HEAA') or 2-hydroxyethylmethacrylate ('HEMA+'), or are HEMA-free ('HEMA-'), to dentin, either measured at 1 week $(1 \mathrm{w})$ or upon 6-month $(6 \mathrm{~m})$ water storage, this when the adhesives were applied in 2SE mode. The black closed dots and the red open squares represent the mean $\mu$ TBS and the fitted linear mixedeffects (LME) means, respectively. The blue thick and orange horizontal lines within each box represents the $1 \mathrm{w}$ and $6 \mathrm{~m} \mu$ TBS median, respectively. Statistically significant differences between experimental groups according to the LME model are indicated by specific symbols and explained in the graph legend at the top-right side.

Fig. 4 Bar graph presenting the light-microscopy failure-analysis data for the experimental 'IMMEDIATE' (left) and aged '6-MONTH' specimens (right), either applied in E\&R (top) or SE mode (bottom). D: Dentin; I: Interfacial; M: Mixed; C: Composite.

Fig. 5 SEM failure analysis of the DiEthyl AcrylAmide ('DEAA') experimental adhesive applied in 3E\&Ra (a-d) and 2SEa (e-h) mode at 1 week (IMMEDIATE: a,b and e,f) and upon additional 6-month water storage (AGED: c,d and g,h), and of the HydroxyEthyl AcrylAmide ('HEAA') experimental adhesive applied in 3E\&Ra (A-D) and 2SEa (E-H) mode at 1 week (IMMEDIATE: A,B and E,F) and upon additional 6-month water storage (AGED: C,D and G,H). (a,b: DEAA_3E\&R_1w) Representative SEM photomicrographs of an 'interfacial failure', observed at the dentin side in (a), with high magnification of the white square in (b), showing the top of the hybrid layer. (c,d: DEAA_3E\&R_6m) Representative SEM photomicrographs of a 'mixed failure', observed at the dentin side in (d), with high magnification 
of the white rectangular in (d1), showing both fractured dentin and the top of the hybrid layer, the latter with ultra-high magnification in (d2). (e,f: DEAA_2SE_1w) Representative SEM photomicrographs of an 'interfacial failure', observed at the dentin side in (e), with high magnification of the white square showing dentinal tubules and the top of the hybrid layer in (f). (g,h: DEAA_2SE -6m) Representative SEM photomicrographs of an 'interfacial failure', observed at the dentin side in $(g)$, with high magnification of the white rectangle showing the hybrid layer top and dentin in (h). (A,B: HEAA_3E\&R_1w) Representative SEM photomicrographs of an 'interfacial failure', observed at the dentin side with a small fractured composite fragment at the lower left corner in $(A)$, with high magnification of the white square in (B), showing the top of the hybrid layer. (C,D: HEAA_3E\&R_6m) Representative SEM photomicrographs of a 'mixed failure', observed at the dentin side, showing fractured dentin, the top of the hybrid layer and a partially attached adhesive layer in (C), with high magnification of the white square showing resin tags, adhesive, and a fractured dentin part in (D). (E,F: HEAA_2SE_1w) Representative SEM photomicrographs of an 'interfacial failure', observed at the dentin side, showing the top of the hybrid layer in (E), with high magnification of the white square showing clearly the top of the hybrid layer in (F). (G,H: DEAA_2SE _6m) Representative SEM photomicrographs of a 'mixed failure', observed at the dentin side, showing a fractured dentin part in (G), with high magnification of the white rectangle showing the top of the hybrid layer, adhesive and dentin in (H). Ad: Adhesive resin; Co: Composite resin; De: Dentin; HL: Hybrid layer; Rt: Resin tag.

Fig. 6 SEM failure analysis of the 2-HydroxyEthylMethAcrylate (HEMA)-free ('HEMA-') experimental adhesive applied in 3E\&Ra (a-d) and 2SEa (e-h) mode at 1 week (IMMEDIATE: a,b and e,f) and upon additional 6-month water storage (AGED: c,d and g,h), and of the 2-HydroxyEthylMethAcrylate ('HEMA+') experimental adhesive applied in 3E\&Ra (A-D) and 2SEa (E-H) mode at 1 week (IMMEDIATE: $A, B$ and $E, F)$ and upon additional 6-month water storage (AGED: $C, D$ and $G, H)$. (a,b: HEMA-_3E\&R_1w) Representative SEM photomicrographs of a 'mixed failure', observed at the dentin side, with the adhesive partially attached in (a), with high magnification of the white square showing fractured dentin, the top of the hybrid layer and adhesive in (b). (c,d: HEMA-_3E\&R_6m) Representative SEM photomicrographs of an 'interfacial failure', observed at the dentin side, showing the top of the hybrid layer and a partially attached adhesive layer in (c), with high magnification of the white square showing resin tags and the top of the hybrid layer in (d). (e,f: HEMA-_2SE_1w) Representative SEM photomicrographs of a 'mixed failure', observed at the dentin side in (e), with high magnification of the white square showing fractured dentin and the top of the hybrid layer in (f). (g,h:HEMA-_2SE_6m) Representative SEM photomicrographs of an 'interfacial failure', observed at the dentin side, showing a partially attached adhesive layer in (g) with high magnification of the white rectangle showing the top of the hybrid layer, adhesive and dentin in (h). (A,B: HEMA+_3E\&R_1w) Representative SEM 
photomicrographs of an 'interfacial failure', observed at the dentin side, showing a small fractured dentin part with the adhesive partially attached in (A), with higher magnification of the white rectangle showing fractured dentin and the top of the hybrid layer in (B1), with ultra-high magnification of the white rectangle showing the top of the hybrid layer and resin tags in (B2). (C,D: HEMA+_3E\&R_6m) Representative SEM photomicrographs of a 'mixed failure', observed at the dentin side, showing fractured composite, the top of the hybrid layer and a partially attached adhesive layer to dentin in (C), with high magnification of the white square showing fractured composite and a partially attached adhesive layer in (D). (E,F: HEMA+_2SE_1w) Representative SEM photomicrographs of an 'interfacial failure', observed at the dentin side in $(E)$, with high magnification of the white square showing dentin and the top of the hybrid layer in (F). (G,H: HEMA+_2SE_6m) Representative SEM photomicrographs of an 'interfacial failure', observed at the dentin side in (G), with high magnification of the white square showing dentin and the top of the hybrid layer in $(\mathrm{H})$. Ad: Adhesive resin; Co: Composite resin; De: Dentin; HL: Hybrid layer; Rt: Resin tag.

Fig. 7 TEM photomicrographs of adhesive-dentin interfaces when the DEAA adhesive-resin formulation was bonded to dentin following a 3E\&R (a-d) and 2SE (A-D) bonding mode. (a-d: DEAA_3E\&R) (a) Nondemineralized, unstained overview section, showing a tight interfacial interaction of DEAA applied in 3E\&R mode at dentin with the formation of a thick (4-6 $\mu \mathrm{m}$ ) hybrid layer. (b) Higher magnification of the white square in (a), detailing the hydroxyapatite-free hybrid layer along with a tubule orifice, in which a resin tag was formed. (c) Magnification of the small white square in (b), showing a thin layer of partially demineralized dentin at the transition to unaffected dentin. (d) High magnification of the large white square in (b), showing interfacial nano-layering (black hand pointer) within the resin tag at the tubule orifice. (A-D: DEAA_2SE) (A) Non-demineralized, unstained overview section, showing a tight interfacial interaction of DEAA applied in 2SE mode at dentin with the formation of a hybrid layer that is hardly observable. (B) Higher magnification of the white square in $(A)$, showing the thin $(<1 \mu \mathrm{m})$ hybrid layer being rich in hydroxyapatite. (C) Magnification of the white square in (B), detailing the hybrid layer with partially demineralized dentin gradual transitioning into unaffected dentin. (D) High magnification of the white square in (C), disclosing interfacial nano-layering (black hand pointer) within the adhesive resin immediately above the submicron hybrid layer.

Fig. 8 TEM photomicrographs of adhesive-dentin interfaces when the HEAA adhesive-resin formulation was bonded to dentin following a 3E\&R (a-d) and 2SE (A-D) bonding mode. (a-d: HEAA_3E\&R) (a) Nondemineralized, unstained overview section, showing a tight interfacial interaction of HEAA applied in 3E\&R mode at dentin with the formation of a thick (4-6 $\mu \mathrm{m})$ hybrid layer. (b) Higher magnification of the white square in (a), detailing the hydroxyapatite-free hybrid layer aside a tubule orifice, in which a 
resin tag was formed. (c) Magnification of the large white square in (b), showing tubule wall hybridization with an abrupt transition to unaffected dentin. (d) High magnification of the small white square in (b), disclosing interfacial nano-layering (black hand pointer) within the resin tag at the tubule orifice. (A-D: HEAA_2SE) (A) Non-demineralized, unstained overview section, showing a tight interfacial interaction of HEAA applied in 2SE mode at dentin with the formation of a hybrid layer that is hardly observable. (B) Higher magnification of the white square in (A), showing the thin $(<1 \mu \mathrm{m})$ hybrid layer being rich in hydroxyapatite, along with an obliquely sectioned tubule orifice, in which a resin tag was formed. (C) Magnification of the white square in (B), detailing the hybrid layer with partially demineralized dentin gradual transitioning into unaffected dentin. (D) High magnification of the white square in (C), disclosing interfacial nano-layering (black hand pointer) within the adhesive resin immediately above the submicron hybrid layer.

Fig. 9 TEM photomicrographs of adhesive-dentin interfaces when the HEMA- adhesive-resin formulation was bonded to dentin following a 3E\&R (a-d) and 2SE (A-D) bonding mode. (a-d: HEMA_3E\&R) (a) Non-demineralized, unstained overview section, showing a tight interfacial interaction of HEMA- applied in 3E\&R mode at dentin with the formation of a thick (4-6 $\mu \mathrm{m})$ hybrid layer. (b) Higher magnification of the white square in (a), detailing the hydroxyapatite-free hybrid layer along with the beginning of a resin tag at a tubule orifice. (c) Magnification of the white square in (b), showing the hydroxyapatite-free hybrid layer along with tubule wall hybridization with a relatively abrupt transition to unaffected dentin. (d) High magnification of the small white square in (b), disclosing interfacial nanolayering (black hand pointer) near a resin tag at the tubule orifice. (A-D: HEMA-_2SE) (A) Nondemineralized, unstained overview section, showing a tight interfacial interaction of HEMA- applied in 2SE mode at dentin with the formation of a hybrid layer that is hardly observable. (B) Higher magnification of the white square in $(A)$, detailing the thin $(<1 \mu \mathrm{m})$ hybrid layer being rich in hydroxyapatite. (C) Magnification of the white square in (B), disclosing interfacial nano-layering within the adhesive resin immediately above the submicron hybrid layer. (D) High magnification of the black square in (C), detailing interfacial nano-layering (black hand pointers) within the adhesive-resin layer.

Fig. 10 TEM photomicrographs of the interface of the HEMA+ adhesive-resin formulation bonded to dentin when applied in 3E\&R (a-d) and 2SE (A-D) bonding mode. (a-d: HEMA+_3E\&R) (a) Nondemineralized, unstained overview section, showing a tight interfacial interaction of HEMA+ applied in 3E\&R mode at dentin; with the formation of a thick (4-6 $\mu \mathrm{m}$ ) hybrid layer. (b) Higher magnification of the white square in (a), detailing the hydroxyapatite-free hybrid layer along with the beginning of two resin tags at two obliquely sectioned tubule orifices. (c) Magnification of the large white square in (b), showing partially demineralized dentin at the tubule orifice gradually transitioning to unaffected 
dentin. (d) High magnification of the small white square in (b), disclosing interfacial nano-layering (black hand pointer) immediately above the hybrid layer. (A-D: HEMA+_2SE) (A) Non-demineralized, unstained overview section, showing a tight interfacial interaction of HEMA+ applied in 2SE mode at dentin with the formation of a hybrid layer that is hardly observable. (B) Higher magnification of the white rectangle in $(A)$, detailing the thin $(<1 \mu \mathrm{m})$ hybrid layer, which is rich in hydroxyapatite. (C) Magnification of the white square in (B), disclosing interfacial nano-layering within the adhesive resin immediately above the submicron hybrid layer. (D) High magnification of the white square in (C), detailing interfacial nano-layering (black hand pointer) within the adhesive-resin layer.

Fig. 11 Collage of graphs presenting the flexural strength (FS) of the four experimental adhesive-resin formulations in (a), with FS measured at 24 hours ('24h'; blue) and after 6-month storage in distilled water (' $6 \mathrm{~m}$ '; orange), the contact angle (CA) in (b), representing surface wetting of the four experimental adhesive-resin formulations on primed dentin, the viscosity of the four experimental adhesive-resin formulations in (c), and the watersorption of the four experimental adhesive-resin formulations in (d). Statistical difference between experimental groups, as determined by one-way ANOVA, are indicated by different letters in the grey bar above each graph. 
Fig. 1

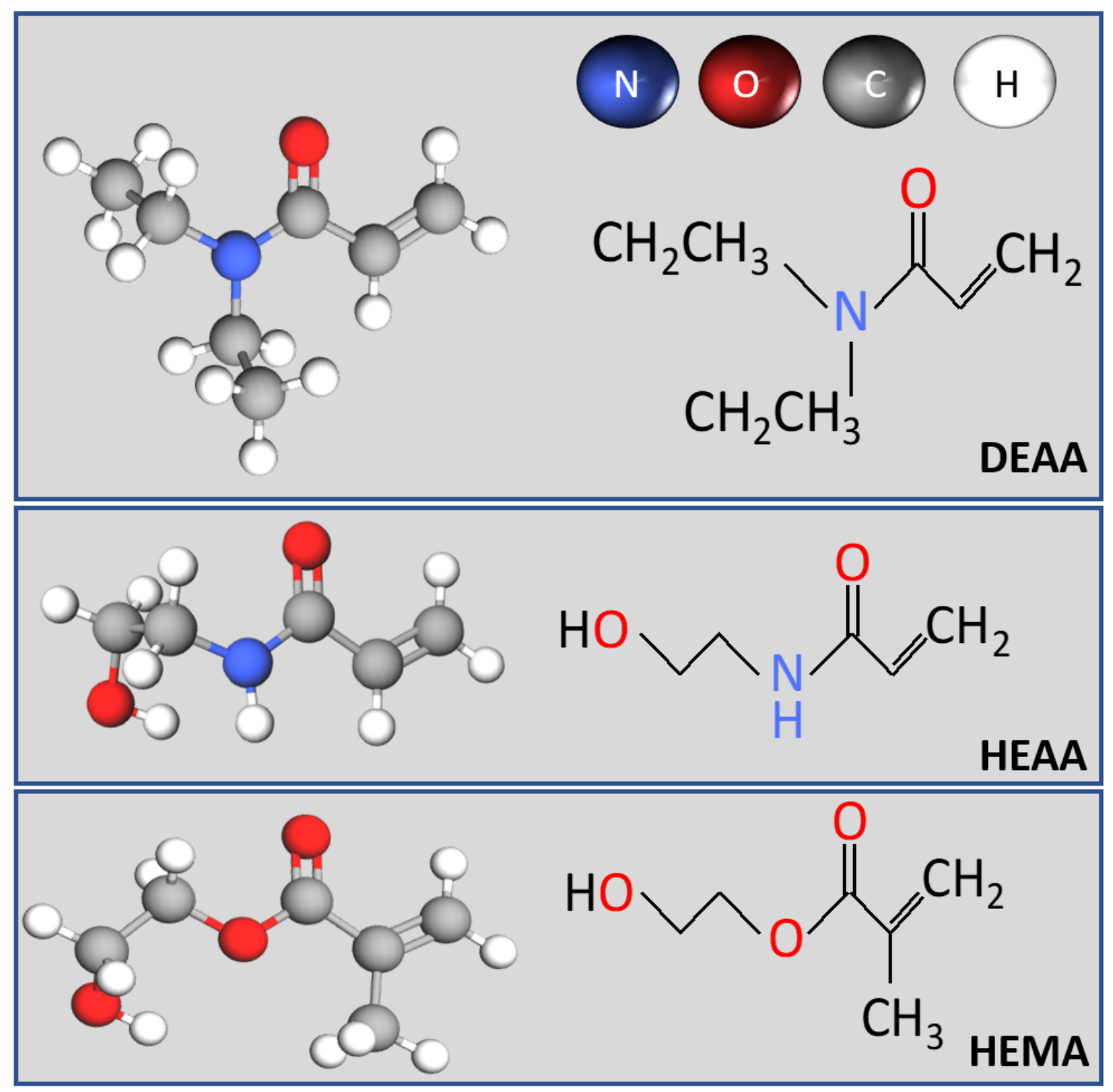


Fig. 2

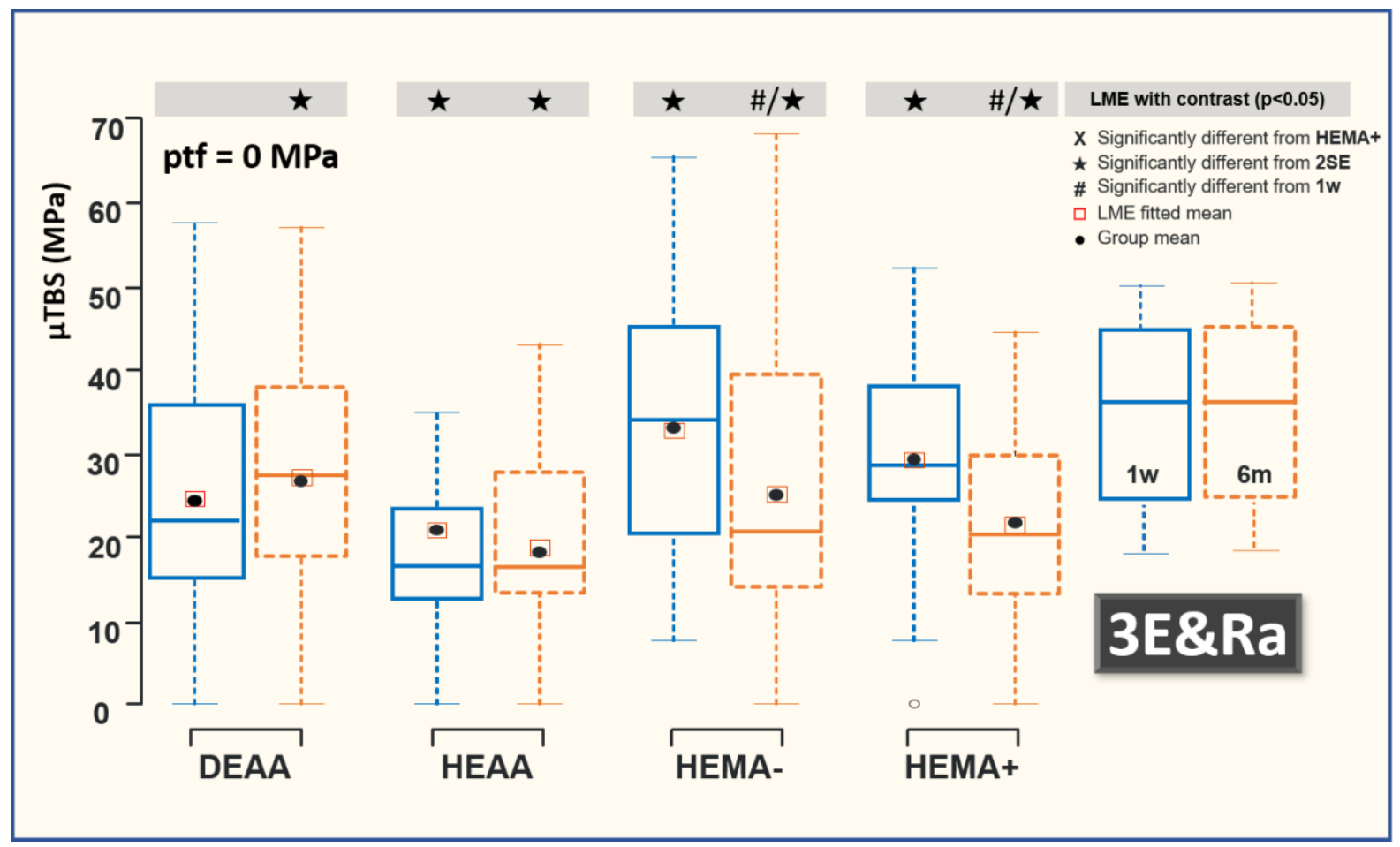


Fig. 3

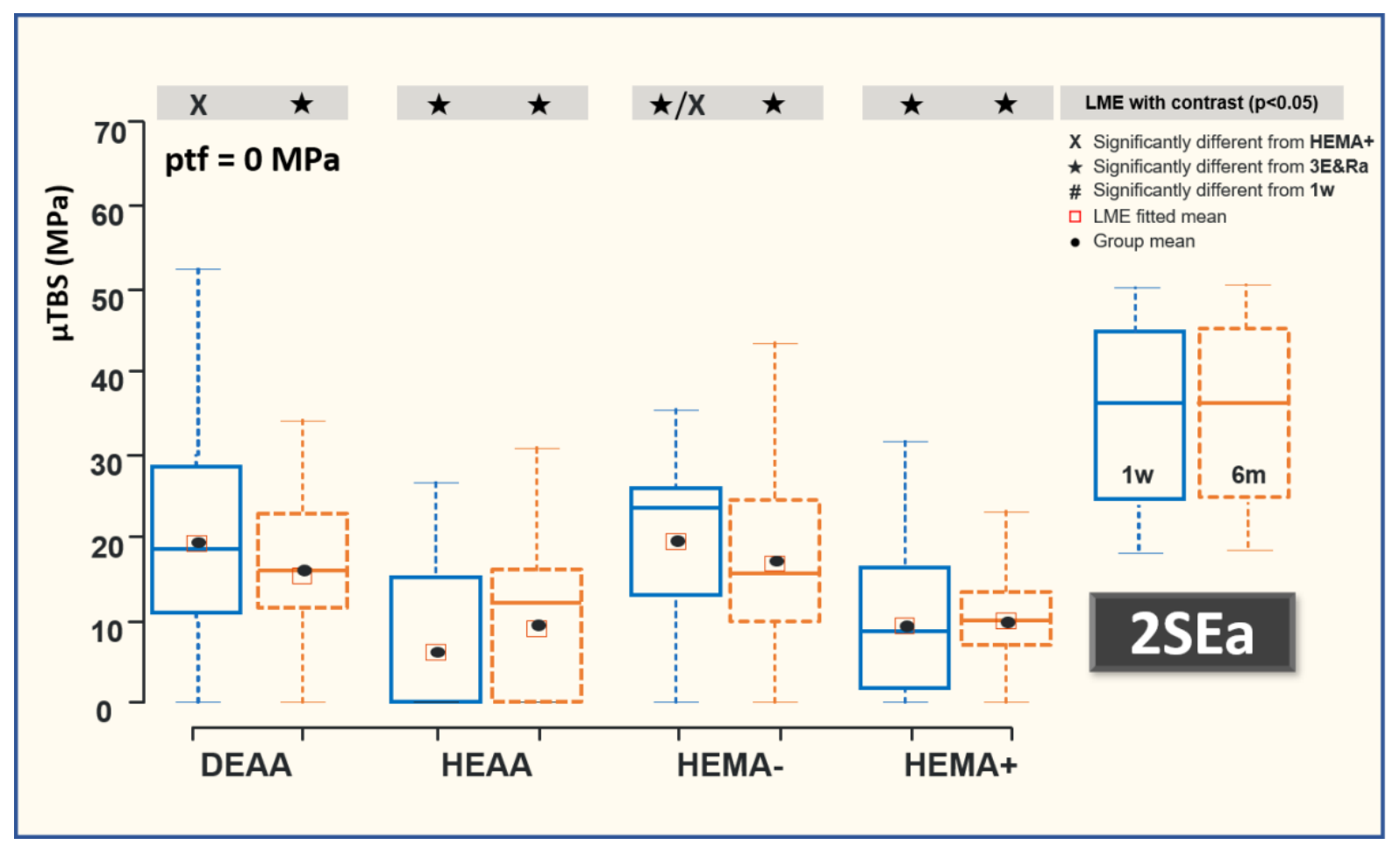


Fig. 4

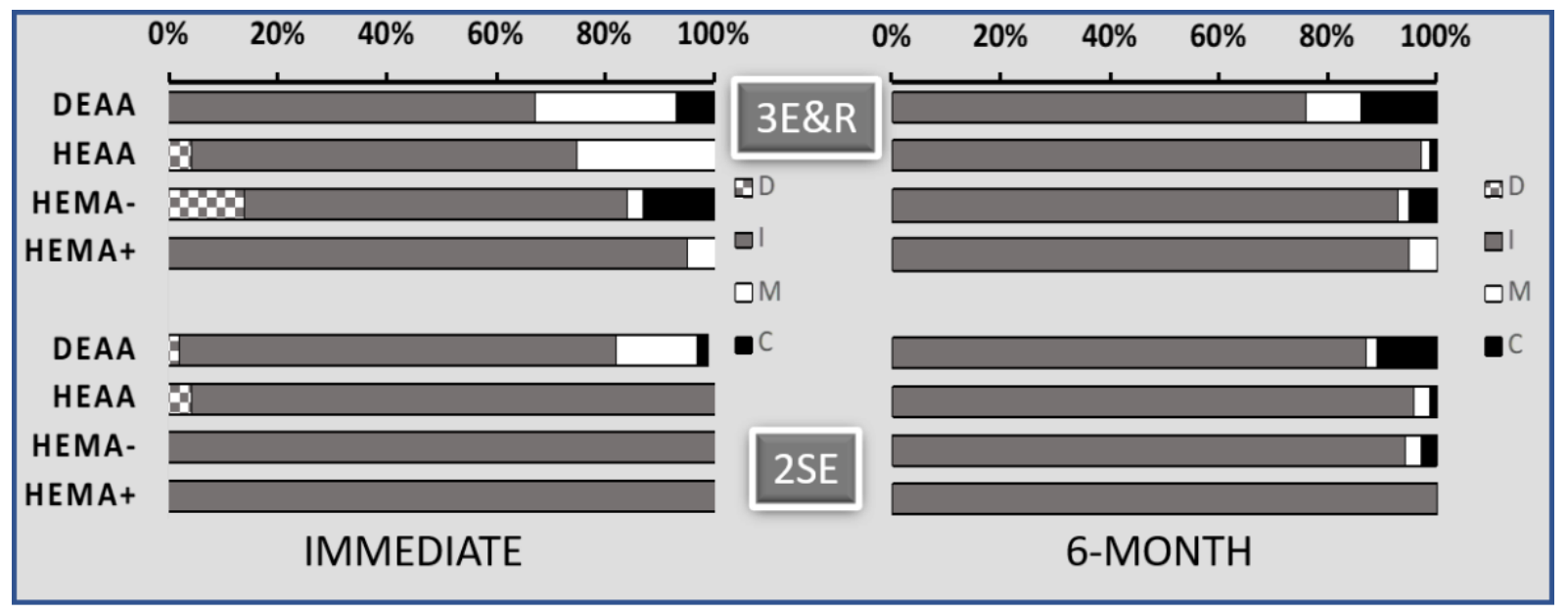


Fig. 5
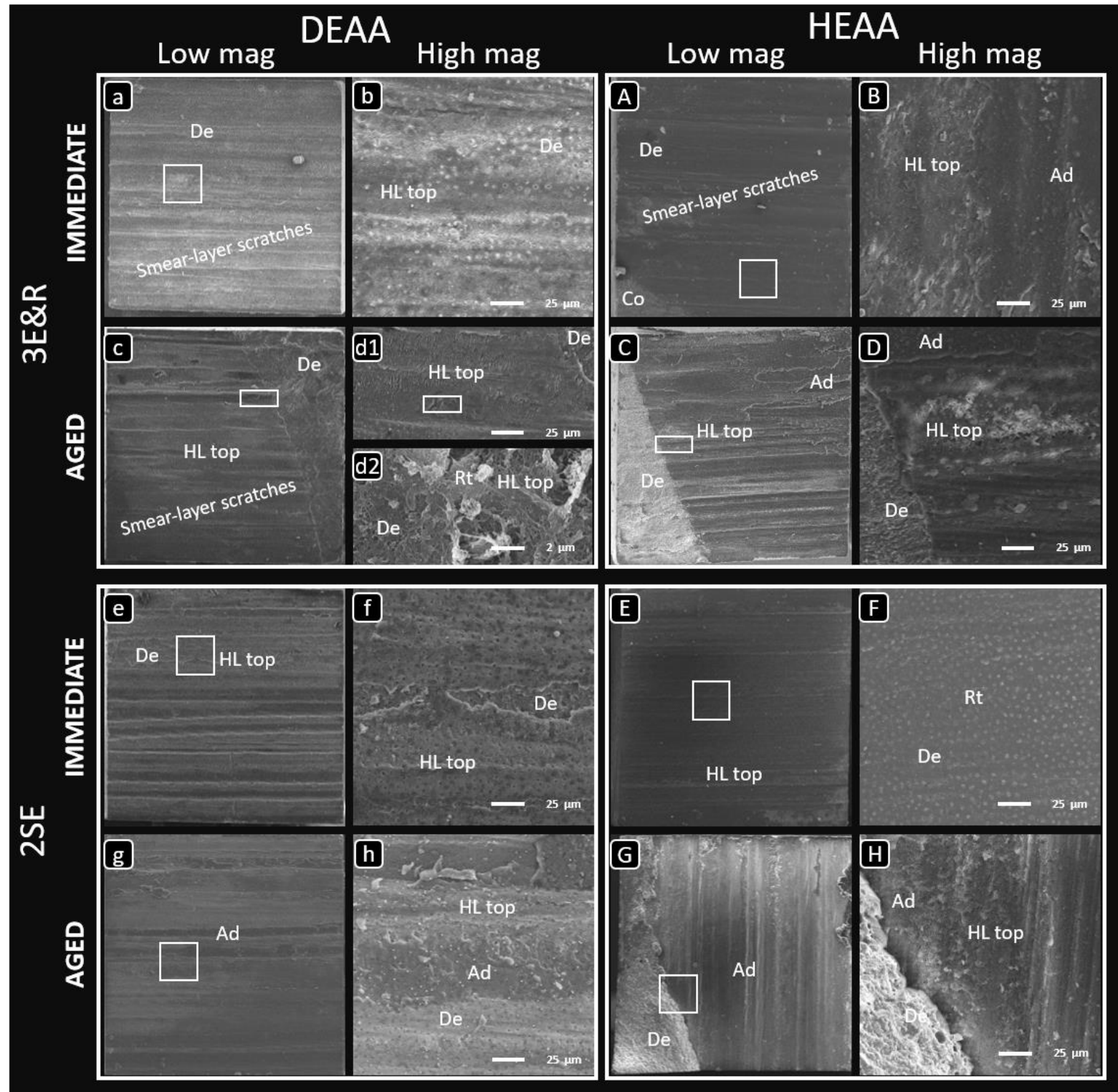
Fig. 6
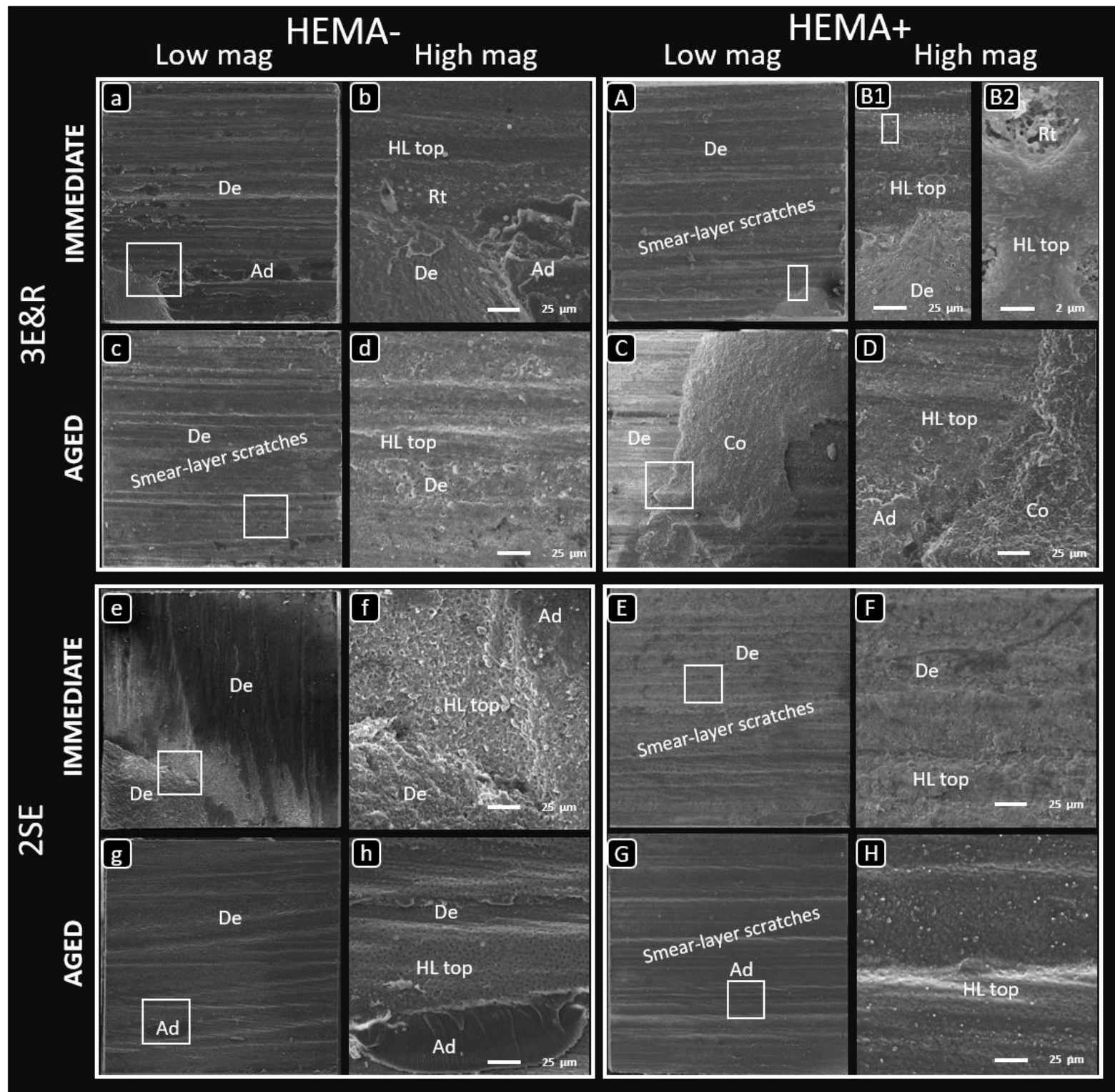
Fig. 7

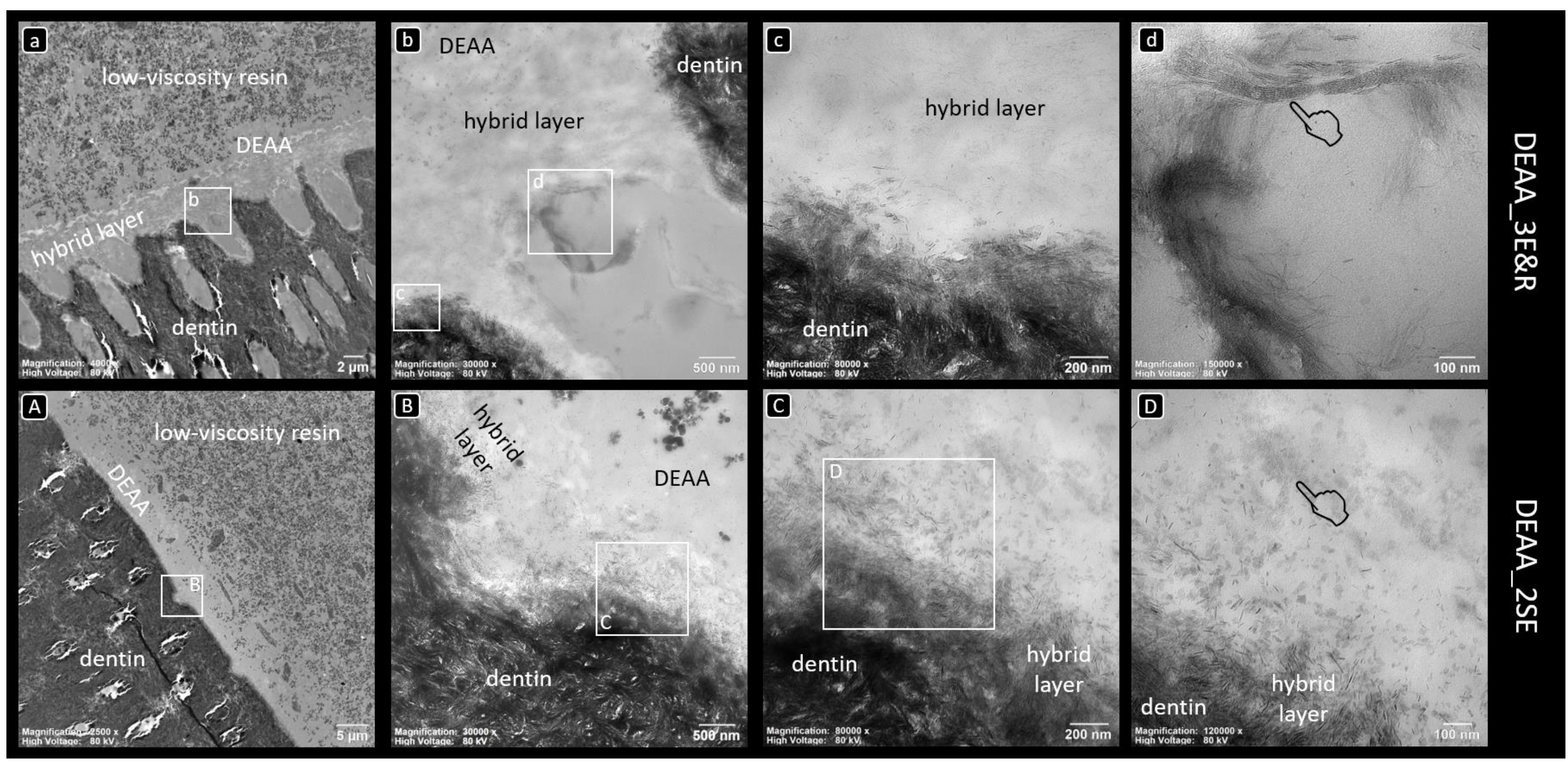


Fig. 8

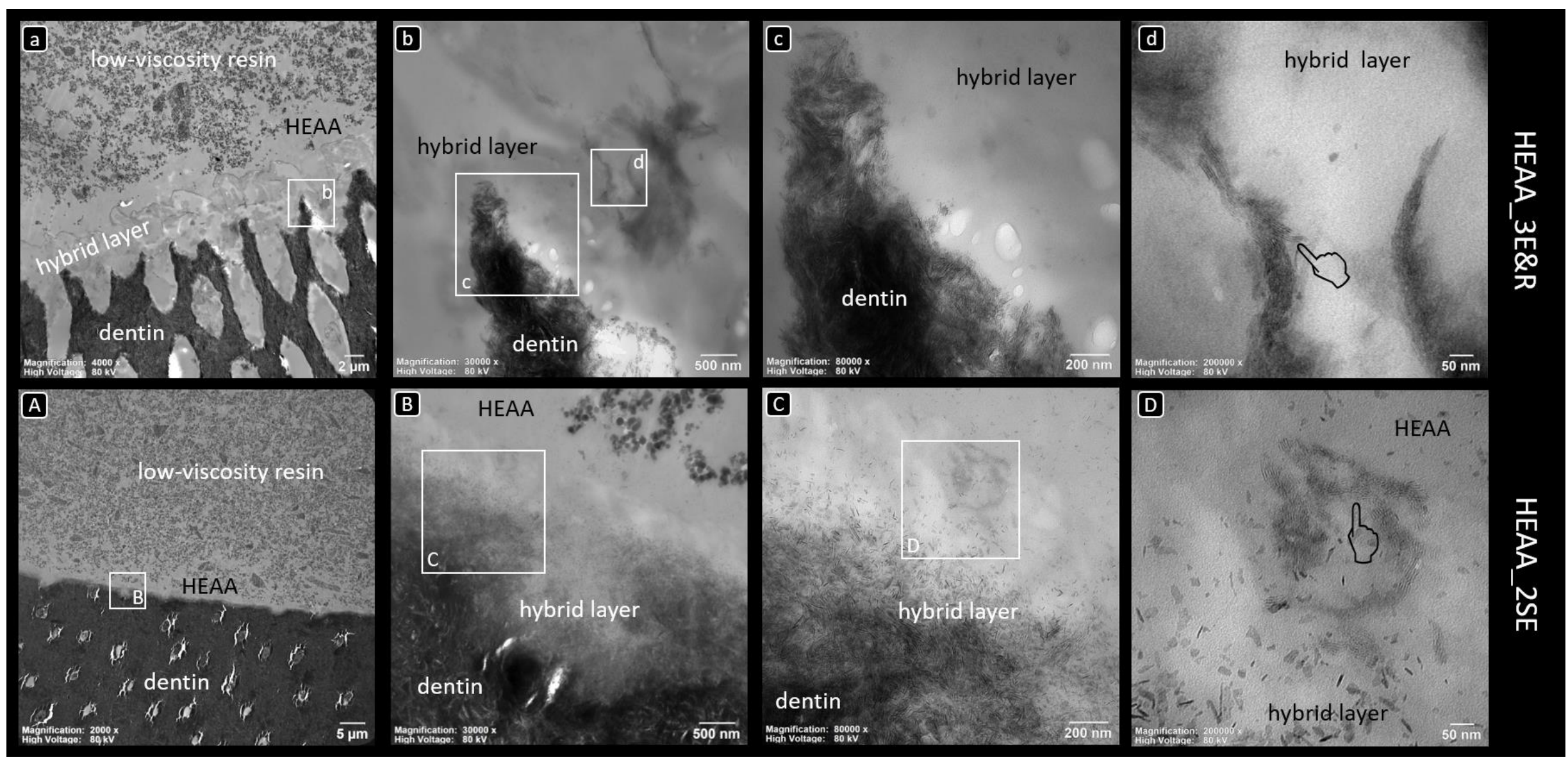


Fig. 9

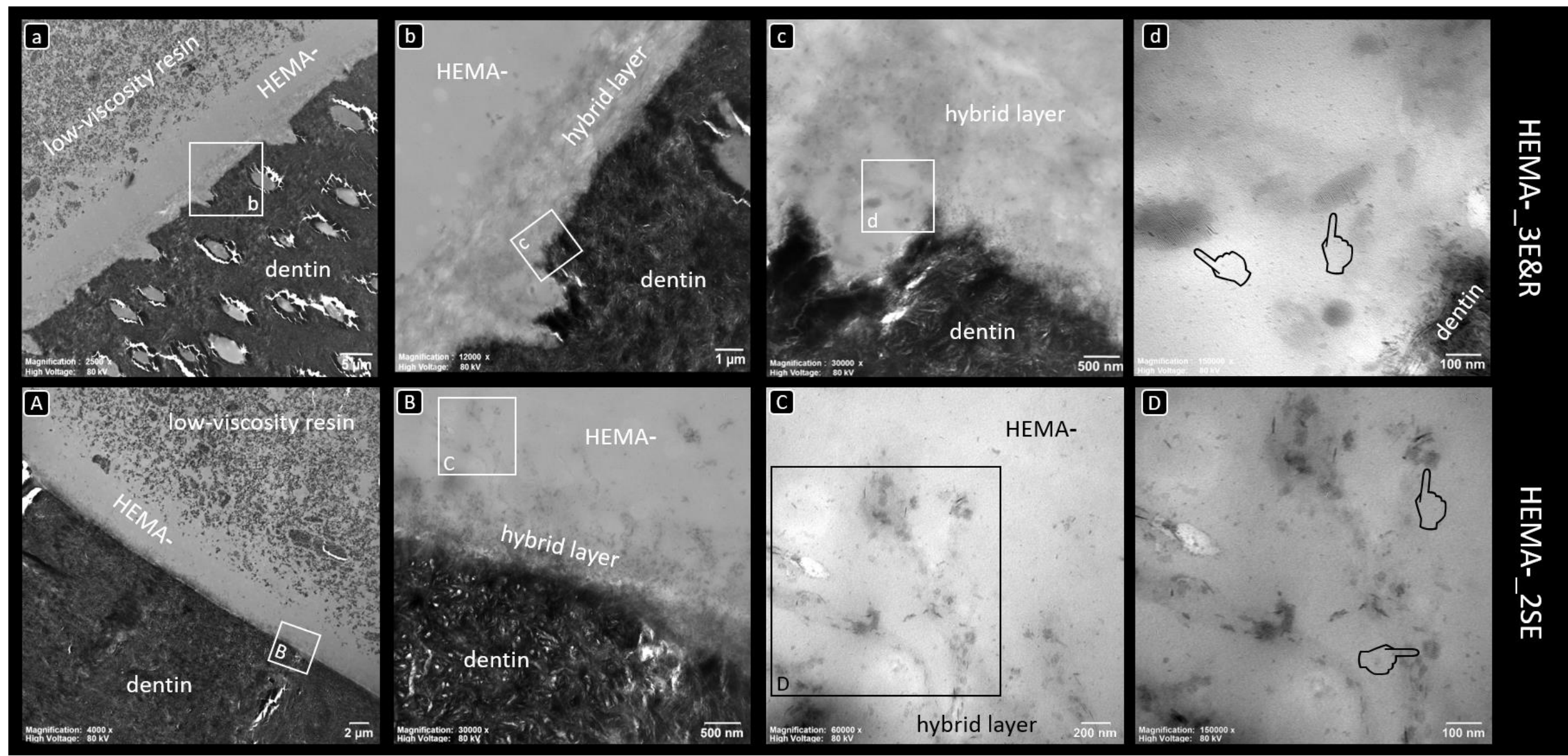


Fig. 10

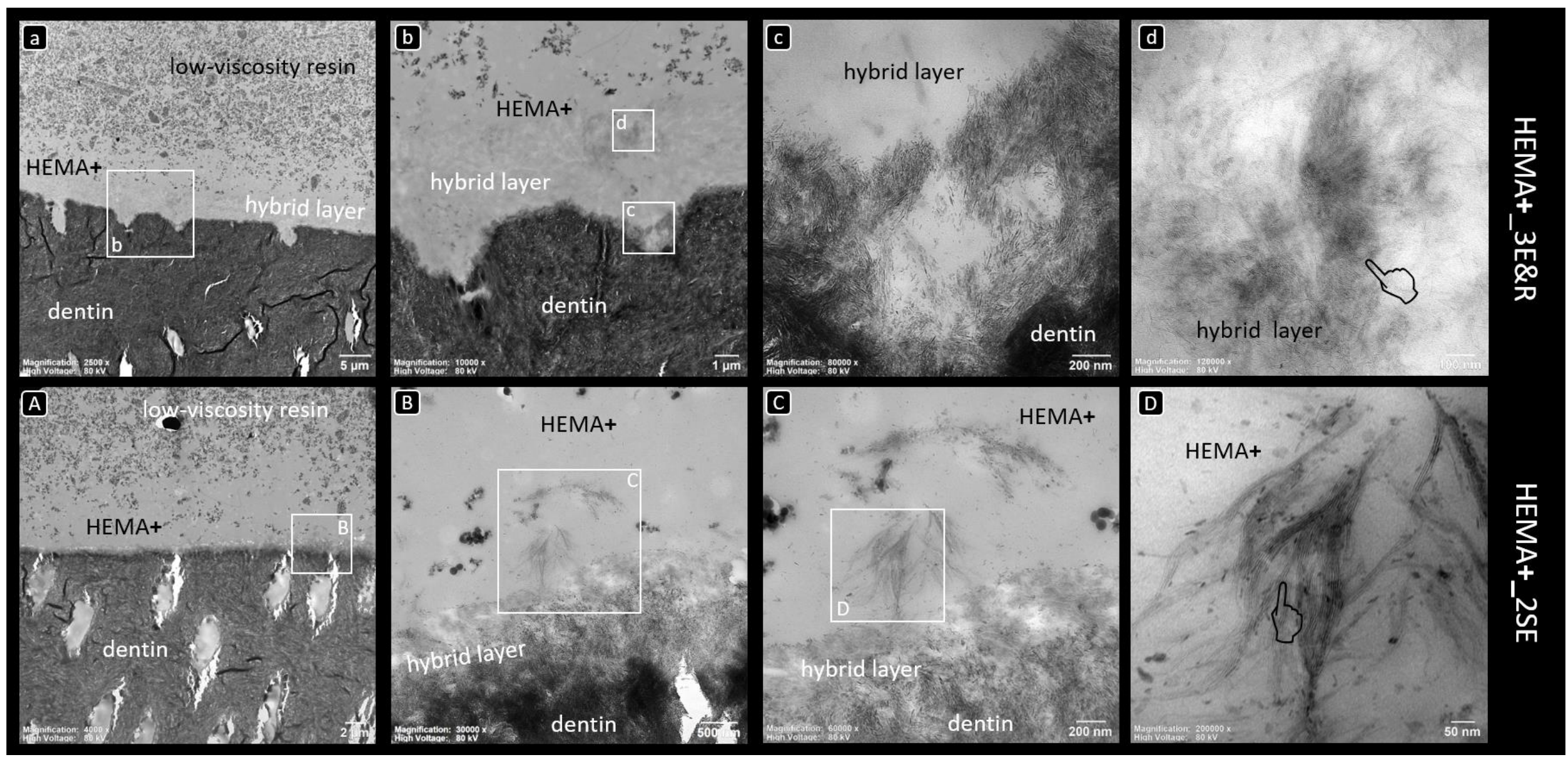


Fig. 11

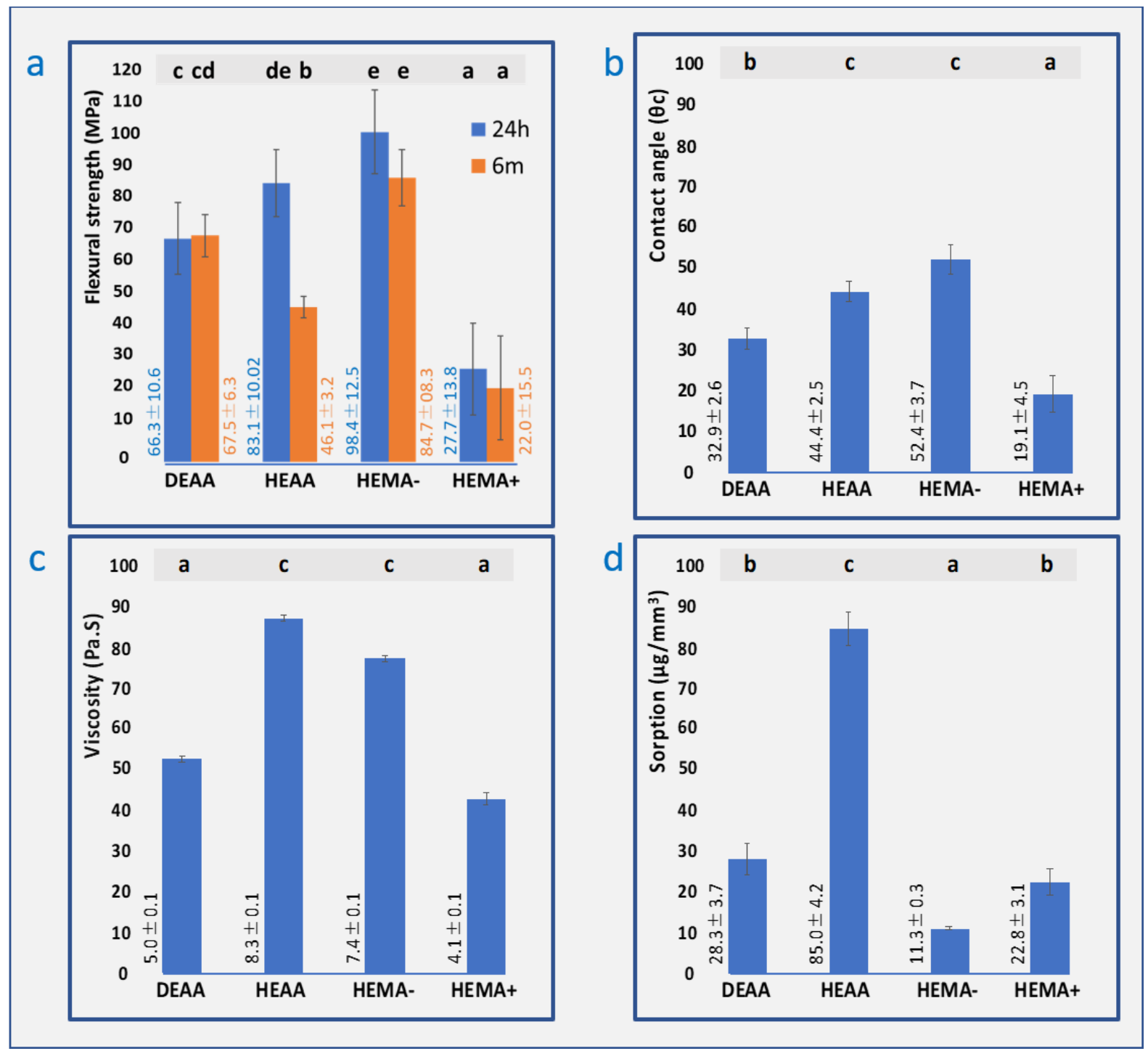

